\title{
Shedding light on a dark state: The energetically lowest quintet state of $\mathbf{C}_{\mathbf{2}}$
}

\author{
P. Bornhauser, Y. Sych, G. Knopp, T. Gerber, and P. P. Radia) \\ Paul Scherrer Institute, General Energy Department, CH-5232 Villigen, Switzerland
}

(Received 14 October 2010; accepted 21 November 2010; published online 24 January 2011)

\begin{abstract}
In this work we present a deperturbation study of the $d^{3} \Pi_{g}, v=6$ state of $\mathrm{C}_{2}$ by double-resonant four-wave mixing spectroscopy. Accurate line positions of perturbed transitions are unambiguously assigned by intermediate level labeling. In addition, extra lines are accessible by taking advantage of the sensitivity and high dynamic range of the technique. These weak spectral features originate from nearby-lying dark states that gain transition strength through the perturbation process. The deperturbation analysis of the complex spectral region in the $(6,5)$ and $(6,4)$ bands of the Swan system $\left(d^{3} \Pi_{g}-a^{3} \Pi_{u}\right)$ unveils the presence of the energetically lowest high-spin state of $\mathrm{C}_{2}$ in the vicinity of the $d^{3} \Pi_{g}, v=6$ state. The term energy curves of the three spin components of the $d$ state cross the five terms of the $1{ }^{5} \Pi_{g}$ state at rotational quantum numbers $N \leq 11$. The spectral complexity for transitions to the $v=6$ level of $d^{3} \Pi_{g}$ state is further enhanced by an additional perturbation at $N=19$ and 21 owing to the $b^{3} \Sigma_{g}^{-}, v=19$ state. The spectroscopic characterization of both dark states is accessible by the measurement of 122 "window" levels. A global fit of the positions to a conventional Hamiltonian for a linear diatomic molecule yields accurate molecular constants for the quintet and triplet perturber states for the first time. In addition, parameters for the spin-orbit and $L$-uncoupling interaction between the electronic levels are determined. The detailed deperturbation study unravels major issues of the so-called high-pressure bands of $\mathrm{C}_{2}$. The anomalous nonthermal emission initially observed by Fowler in 1910 [Mon. Not. R. Astron. Soc. 70, 484 (1910)] and later observed in numerous experimental environments are rationalized by taking into account "gateway" states, i.e., rotational levels of the $d^{3} \Pi_{g}, v=6$ state that exhibit significant ${ }^{5} \Pi_{g}$ character through which all population flows from one electronic state to the other. @ 2011 American Institute of Physics. [doi:10.1063/1.3526747]
\end{abstract}

\section{INTRODUCTION}

The Swan band emission between 400 and $700 \mathrm{~nm}$ is a prominent feature in all carbon-containing flames. In the interstellar medium dicarbon molecules have been detected in diffuse and translucent clouds, in the circumstellar shell of carbon stars, and in comets as well and they play an important role in the formation of hydrogen-deficient carbon molecules and the formation of cyclic molecules. ${ }^{1,2}$ The intense $d^{3} \Pi_{g}-a^{3} \Pi_{u}$ electronic transitions are used widely to detect the molecule in combustion ${ }^{3-11}$ and astronomy [Ref. 12 and references therein] studies to characterize and test chemical mechanisms. The quantitative interpretation of spectra requires precise molecular constants for the computation of the complex molecular spectra of $\mathrm{C}_{2}$.

Starting with the pioneering works of Wollaston in 1802 (Ref. 13) and Swan in 1857 (Ref. 14) a very large number of publications have been presented on the spectroscopy of $\mathrm{C}_{2}$. Summaries of the research can be found in Refs. 12, 15-17 and references therein. However, despite the fact that the $d^{3} \Pi_{g}-a^{3} \Pi_{u}$ system of $\mathrm{C}_{2}$ has been studied for over two centuries, a complete rotational analysis has not been achieved. The most recent comprehensive investigation of the Swan system has been performed by applying Fourier transform spectroscopy. ${ }^{17} \Delta v=-3$ to $\Delta v=+2$

${ }^{a)}$ Electronic mail: peter.radi@psi.ch. sequence bands in the $14000-24000 \mathrm{~cm}^{-1}(\approx 410-710 \mathrm{~nm})$ spectral range have been investigated. A global fit to assigned rotational transitions of 30 bands has been used to derive spectroscopic constants. However, these molecular constants are not sufficiently complete to model all features of the $d^{3} \Pi_{g}-a^{3} \Pi_{u}$ system because of local perturbations in many vibrational levels. Difficulties arise mainly due to the interaction of the Swan levels with several neighboring electronic states. In Fig. 1 the low-lying vibronic origins of the singlet, triplet, and quintet manifold are indicated relative to the ground $X^{1} \Sigma_{g}^{+}, v^{\prime \prime}=0$ state. Please note that the $1^{5} \Pi_{g}$ is measured and characterized in this work for the first time to the best of our knowledge (vide infra).

The occurrence of perturbations in the dicarbon molecule is well known. Ballik and Ramsay ${ }^{18}$ reported perturbations in the $b^{3} \Sigma_{g}^{-}$state by vibrational levels of the $X^{1} \Sigma_{g}^{+}$state and deduced from the spin-forbidden interaction that the perturbing state lies $610 \mathrm{~cm}^{-1}$ below the $a$ state and established it as the ground state of the $\mathrm{C}_{2}$ molecule. Perturbations in $v=0-2$ levels of the upper Swan $d^{3} \Pi_{g}$ state have been observed subsequently by Callomon and Gilby ${ }^{19}$ and the perturbing levels have been identified as belonging to the vibrational levels $v=11-16$ of the $b^{3} \Sigma_{g}^{-}$electronic state. Additional perturbations in the $v=3-5$ vibrational levels of the $d^{3} \Pi_{g}$ state have been reported by Phillips. ${ }^{20}$ It has been shown that the ground state $X^{1} \Sigma_{g}^{+}$is involved in certain perturbations. Other perturbations in the $v=4$ and 5 levels were 


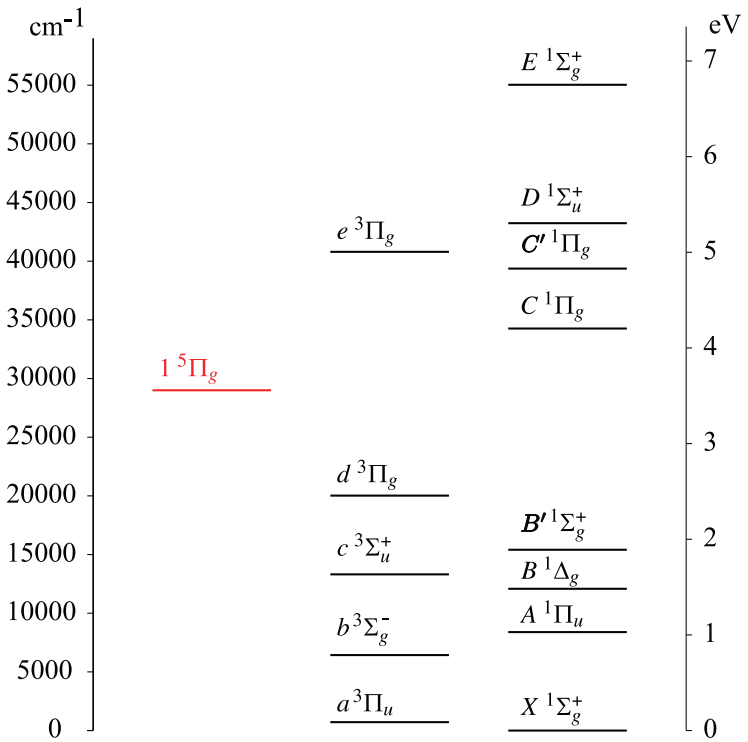

FIG. 1. Low-lying electronic states of $\mathrm{C}_{2}$. The vibronic origins of the singlet, triplet, and quintet manifolds are indicated relative to $X^{1} \Sigma_{g}^{+}, v=0$ state.

suggested to occur due to the a ${ }^{1} \Delta_{g}$ and/or ${ }^{1} \Sigma_{g}^{+}$state. These two states labeled $B$ and $B^{\prime}$, respectively, were discovered only many years later by Douay et al. ${ }^{21}$ applying Fourier transform emission spectroscopy in hydrocarbon discharges. The perturbations of the $d^{3} \Pi_{g}, v=0$ level by the $B^{1} \Delta_{g}$ and $b^{3} \Sigma_{g}^{-}$states were confirmed by Amiot. ${ }^{22}$ Numerous perturbations were found for $v=0,1,2,4,6,8,9$, and 10 by the Fourier transform emission spectroscopy investigation of Tanabashi et al. ${ }^{17}$ mentioned above but details could not be analyzed satisfactorily from the complex and overlapping band structure. In addition, disagreement for a number of the reported perturbations for the $v=4$ level have been found in a recent investigation of the $d^{3} \Pi_{g}-c^{3} \Sigma_{u}^{+}$system by Joester et al. ${ }^{23}$

Very recently, we have applied double-resonance spectroscopy by two-color resonant four-wave mixing (TCRFWM) for the unambiguous assignments of the perturbed levels in $v=4$ by intermediate level labeling. ${ }^{24}$ Several line positions found in the literature had to be reassigned. Additionally, by taking advantage of the high dynamic range resulting in substantial S/N ratios (up to $10^{9}$ for the strongest Swan bands), extra transitions could be observed and assigned in a straightforward manner to the perturbing $b^{3} \Sigma_{g}^{-}, v=16$ electronic state. Significantly perturbed rotational transitions in the $(4,3)$ band [vibrational bands are denoted by convention as $\left.\left(v^{\prime}, v^{\prime \prime}\right)\right]$ of the Swan system were reported for the first time. A least-squares fit to 35 perturbed and perturbing transitions deduced the vibronic origin, rotational, and spinspin coupling constant of the $b^{3} \Sigma_{g}^{-}, v=16$ state as well as the spin-orbit and $L$-uncoupling parameters for the interaction between the $d^{3} \Pi_{g}, v=4$ and $b^{3} \Sigma_{g}^{-}, v=16$ states.

In this work, the TC-RFWM method is applied to investigate the heavily perturbed $v=6$ vibrational level of the $d^{3} \Pi_{g}$ state. This vibrational level has been shown by Fox and Herzberg ${ }^{25,26}$ to be responsible for the so-called high pressure (HP) bands. The term refers to a strikingly enhanced emis- sion from $v=6$ in respect to other vibrational levels. These bands were initially observed by Fowler in 1910 in a discharge through $\mathrm{CO}$ at relatively high pressure $(\approx 13 \mathrm{kPa}) .{ }^{27}$ The author assigned tentatively spectral features observed in comet spectra to these HP bands and suspected carbon monoxide as emitter. Later, Johnson and Asundi ${ }^{28}$ have established that the HP band emission originates from the $\mathrm{C}_{2}$ molecule. Since then, the HP bands have been observed in numerous experimental environments that have been reviewed by Little and Brown. ${ }^{29}$ The process leading to the enhanced emission from $d^{3} \Pi_{g}, v=6$ has been attributed to the crossing of a nearby-lying electronic state. An investigation by Meinel and Messerle ${ }^{30}$ of perturbed rotational levels suggested that the levels with $N=19$ and 21 are indeed perturbed by the $b^{3} \Sigma_{g}^{-}$state. However, owing to the observation of a striking intensity distribution of perturbed rotational levels with $N \leq 11$ indicating a nonthermal population filling in respect to the depletion of the $N=19$ and 21 levels, a $\Delta_{g}$ perturbing state was tentatively taken into account. By considering the results from an $a b$ initio configuration-interaction study of the valence states of $\mathrm{C}_{2}$ by Kirby and Liu, ${ }^{31}$ the authors of Ref. 29 suggested that the computed metastable ${ }^{5} \Pi_{g}$ state is responsible for the enhanced emission from $v=6$. They proposed that following formation of this metastable state by an atom-atom collision mechanism, population transfer from ${ }^{5} \Pi_{g}$ to $d^{3} \Pi_{g}, v=6$ should take place via collisionally induced curve crossing. The $\mathrm{C}+\mathrm{C}_{2} \mathrm{O} \longrightarrow \mathrm{C}_{2}+\mathrm{CO}$ reaction suggested by several authors ${ }^{32-34}$ as the main contributor of the HP bands has been considered insignificant in this work. On the other hand, Caubet and Dorthe ${ }^{35}$ have emphasized on the basis of their kinetic study of the HP in a fast flow reactor that both reactions, $\mathrm{C}+\mathrm{C}+\mathrm{M}$ and $\mathrm{C}+\mathrm{C}_{2} \mathrm{O}$ were involved. However, an identification of the perturbing state that could provide additional information pertinent to the proposed production pathways has not been achieved up to date.

\section{EXPERIMENT}

The molecular beam apparatus and the slit discharge assembly have been described previously in detail ${ }^{24,36-38}$ and only a brief outline of the experiment is given in the following. Radicals are generated by pulsing the precursor mixture of $\approx 0.5 \%$ acetylene in argon through a $30 \times 1.0 \mathrm{~mm}$ slit-jet nozzle based on the design of Linnartz. ${ }^{39}$ Pressures behind the valve are typically 5-7 atm. A high-voltage pulse of -800 to $-1000 \mathrm{~V}$ is applied to the electrodes by using a precisely timed trigger pulse relative to the valve opening. In comparison with a cylindrical source, the two-dimensional slit-expansion increases the interaction volume of the four-wave mixing beams with the molecular beam and enhances the sensitivity of the method. In fact, our recent experiments on $\mathrm{C}_{2}$ and $\mathrm{HC}_{4} \mathrm{~S}$ radicals demonstrated substantial signal-to-noise ratios and a degenerate four-wave mixing (DFWM) sensitivity among the highest achieved. ${ }^{37}$ The molecular beam propagates into the vacuum chamber and interacts with the laser beams roughly $2 \mathrm{~cm}$ downstream from the discharged slit-jet exit. During operation a pressure of $\approx 10^{-2} \mathrm{~Pa}$ is maintained.

One or two separately pumped dye lasers (narrowscan, Radiant Dyes) are used for DFWM and TC-RFWM. 
A combination of optical components establishes a forward BOXCARS $^{40}$ configuration. ${ }^{41}$ The three input beams pass along the main diagonals of a parallelepiped and cross with a small angle of $\approx 1^{\circ}$. These beams with wavevectors $\vec{k}_{1}, \vec{k}_{2}$, and $\vec{k}_{3}$ generate a signal beam wavevector $\vec{k}_{4}$ along the fourth diagonal. For the double-resonance TC-RFWM experiment, one of the three incident beams $\left(\vec{k}_{2}\right)$ is replaced by a laser beam of different frequency (PROBE beam). The equal frequency input beams $\left(\vec{k}_{1}\right.$ and $\left.\vec{k}_{3}\right)$ are commonly referred to as PUMP beams. The exact direction of the four-wave mixing signal beam is given by the phase-matching condition $\vec{k}_{1}+\vec{k}_{3}=\vec{k}_{2}+\vec{k}_{4}$ and passes through several optical and spatial filters consisting of 50 or $100 \mu \mathrm{m}$ pinholes to obtain a homogeneous intensity distribution over the entire laser beam diameter. The pinholes remove, in addition, interfering scattered light and unwanted fluorescence. Further reduction of stray light is achieved by a spatial filter consisting of a $30 \mathrm{~mm}$ focal length lens and $50 \mu \mathrm{m}$ pinhole in front of the photomultiplier. Typically $20-30$ pulses are averaged per scan step of the dye laser $\left(5 \times 10^{-4} \mathrm{~nm} / \mathrm{step}\right)$ on the oscilloscope and transferred to a PC (labview) for further analysis. The specified bandwidth of both laser systems is $\approx 0.04 \mathrm{~cm}^{-1}$. Absolute wavelength calibration of the dye laser is performed for each scan step by using a wavemeter (High Finesse/Ångstrom, WS6) with a specified absolute accuracy of $\approx 600 \mathrm{MHz}$ $\left(\approx 0.02 \mathrm{~cm}^{-1}\right)$. Pulse energies are adjusted by using a variable attenuator (Newport, M-935-10) such that relevant transitions are not power broadened due to saturation. In fact, the accurate simulation of four-wave mixing spectra requires careful control of the input laser intensity. ${ }^{42-48}$ For this work however, no attempt was made to model the spectral intensity distribution in detail because results are obtained from line positions. Line intensities however are used as a guide for the assignments. For DFWM mixing scans, laser powers were typically a few $\mu \mathrm{J} /$ pulse. For these conditions, the DFWM spectral signal intensities closely matched the calculated absorption spectra (vide infra). To detect the large variations of the oscillator strengths of the perturbed and perturbing transitions by TC-RFWM, the high dynamic range of the spectroscopic technique is exploited ${ }^{37}$ and the quadratic dependence of the four-wave mixing signal on dipole moment is taken into account. Strong transitions in the Swan band system are measured with laser powers in the $\mathrm{nJ} /$ pulse range. Alternatively, by increasing the laser power to the $\mathrm{mJ} /$ pulse range, transitions of weakly perturbed dark states are unveiled and observed with excellent signal-to-noise ratios.

\section{THEORY}

The $1{ }^{5} \Pi_{g}, a^{3} \Pi_{u}, d^{3} \Pi_{g}$, and $b^{3} \Sigma_{g}^{-}$states are modeled by using a conventional Hamiltonian for a linear molecule ${ }^{49}$ implemented in the computer program PGOPHER ${ }^{50}$ with terms omitted as appropriate

$$
\begin{aligned}
\hat{H}= & T+B \hat{\mathbf{N}}^{2}-D \hat{\mathbf{N}}^{4}+A \hat{\mathbf{L}} \cdot \hat{\mathbf{S}}+\frac{A_{D}}{2}\left[\hat{\mathbf{N}}^{2}, \hat{\mathbf{L}} \cdot \hat{\mathbf{S}}\right]_{+} \\
& +\frac{2}{3} \lambda\left(3 \hat{S}_{z}^{2}-\hat{\mathbf{S}}^{2}\right)
\end{aligned}
$$

$$
\begin{aligned}
& +\frac{1}{2} o\left(\hat{S}_{+}^{2} e^{-2 i \phi}+\hat{S}_{-}^{2} e^{+2 i \phi}\right) \\
& -\frac{1}{2} p\left(\hat{N}_{+} \hat{S}_{+} e^{-2 i \phi}+\hat{N}_{-} \hat{S}_{-} e^{+2 i \phi}\right) \\
& +\frac{1}{2} q\left(\hat{N}_{+}^{2} e^{-2 i \phi}+\hat{N}_{-}^{2} e^{+2 i \phi}\right)
\end{aligned}
$$

where $[\hat{O}, \hat{Q}]_{+}=\hat{O} \hat{Q}+\hat{Q} \hat{O}$.

The first term is the vibronic origin followed by the rotational kinetic energy and spin-orbit coupling terms including their corresponding centrifugal distortion terms. The term containing the spin-spin coupling constant $\lambda$ is followed by the three terms determining the $\Lambda$-doubling parameters $o, p$, and $q$.

The Hamiltonian energy matrix elements for the Swan $d^{3} \Pi_{g}$ and $a^{3} \Pi_{u}$ states are given by

$$
\begin{aligned}
\left\langle\Pi_{0}|\mathrm{H}|^{3} \Pi_{0}\right\rangle= & T-A+\left(B-A_{D}\right)(x+2) \\
& -D\left(x^{2}+6 x+4\right)+\frac{2}{3} \lambda \\
& \mp(o+p+q), \\
\left\langle{ }^{3} \Pi_{1}|\mathrm{H}|^{3} \Pi_{1}\right\rangle= & T+B(x+2)-D\left(x^{2}+8 x\right) \\
& -\frac{4}{3} \lambda \mp \frac{1}{2} q x, \\
\left\langle{ }^{3} \Pi_{2}|\mathrm{H}|^{3} \Pi_{2}\right\rangle= & T+A+\left(B+A_{D}\right)(x-2) \\
& -D\left(x^{2}-2 x\right)+\frac{2}{3} \lambda, \\
\left\langle{ }^{3} \Pi_{0}|\mathrm{H}|^{3} \Pi_{1}\right\rangle= & -\sqrt{2 x}\left[B-\frac{1}{2} A_{D}-2 D(x+2)\right. \\
& \left.\mp \frac{1}{2}(p+2 q)\right], \\
\left\langle{ }^{3} \Pi_{0}|\mathrm{H}|^{3} \Pi_{2}\right\rangle= & -\sqrt{x(x-2)}\left(2 D \pm \frac{1}{2} q\right), \\
\left\langle{ }^{3} \Pi_{1}|\mathrm{H}|^{3} \Pi_{2}\right\rangle= & -\sqrt{2(x-2)}\left(B+\frac{1}{2} A_{D}-2 D x\right),
\end{aligned}
$$

where $x=J(J+1)$. The upper and lower signs before $o, p$, and $q$ refer to $e$ and $f$ sublevels for $d^{3} \Pi_{g}$ and to $f$ and $e$ sublevels for the $a^{3} \Pi_{u}$ state, respectively.

For the $1^{5} \Pi_{g}$ state, the energy matrix elements are applied as follows:

$$
\begin{aligned}
& \left\langle{ }^{5} \Pi_{-1}|\mathrm{H}|^{5} \Pi_{-1}\right\rangle=T+B(x+2)-2 A+4 \lambda, \\
& \left\langle{ }^{5} \Pi_{0}|\mathrm{H}|^{5} \Pi_{0}\right\rangle=T+B(x+6)-A-2 \lambda \pm 3 o, \\
& \left\langle{ }^{5} \Pi_{1}|\mathrm{H}|^{5} \Pi_{1}\right\rangle=T+B(x+6)-4 \lambda, \\
& \left\langle{ }^{5} \Pi_{2}|\mathrm{H}|^{5} \Pi_{2}\right\rangle=T+B(x+2)+A-2 \lambda, \\
& \left\langle{ }^{5} \Pi_{3}|\mathrm{H}|^{5} \Pi_{3}\right\rangle=T+B(x-6)+2 A+4 \lambda, \\
& \left\langle{ }^{5} \Pi_{-1}|\mathrm{H}|^{5} \Pi_{0}\right\rangle=-2 B \sqrt{x}, \\
& \left\langle{ }^{5} \Pi_{-1}|\mathrm{H}|^{5} \Pi_{1}\right\rangle= \pm \sqrt{6} o, \\
& \left\langle{ }^{5} \Pi_{0}|\mathrm{H}|^{5} \Pi_{1}\right\rangle=-B \sqrt{6 x}, \\
& \left\langle{ }^{5} \Pi_{1}|\mathrm{H}|^{5} \Pi_{2}\right\rangle=-B \sqrt{6(x-2)}, \\
& \left\langle{ }^{5} \Pi_{2}|\mathrm{H}|{ }^{5} \Pi_{3}\right\rangle=-2 B \sqrt{x-6 .} .
\end{aligned}
$$

The \pm signs before $o$ refer to the $e$ and $f$ sublevels, respectively. 
The Hamiltonian energy matrix elements for the $b^{3} \Sigma_{g}^{-}$ state are given by

$$
\begin{aligned}
\left\langle{ }^{3} \Sigma_{0}^{-}|\mathrm{H}|^{3} \Sigma_{0}^{-}\right\rangle= & \frac{1}{2}(1 \pm 1)[T+B(x+2) \\
& \left.-D\left(x^{2}+8 x+4\right)-\frac{4}{3} \lambda\right], \\
\left\langle{ }^{3} \Sigma_{1}^{-}|\mathrm{H}|^{3} \Sigma_{1}^{-}\right\rangle= & T+B x-D\left[x^{2}+(1 \pm 1) 2 x\right]+\frac{2}{3} \lambda, \\
\left\langle{ }^{3} \Sigma_{0}^{-}|\mathrm{H}|^{3} \Sigma_{1}^{-}\right\rangle= & -(1 \pm 1) \sqrt{x}[B-2 D(x+1)] .
\end{aligned}
$$

Upper signs refer to $e$ levels and lower signs to $f$ levels.

The spin-orbit interaction parameters between the triplet $d^{3} \Pi_{g}, v=6$ and quintet $1^{5} \Pi_{g}$ states are given by

$$
\left\langle{ }^{3} \Pi_{1}\left|\mathrm{H}_{\mathrm{so}}\right|^{5} \Pi_{1}\right\rangle
$$

and the dependent terms

$$
\begin{aligned}
\left\langle{ }^{3} \Pi_{0}\left|\mathrm{H}_{\mathrm{SO}}\right|^{5} \Pi_{0}\right\rangle & =\left\langle{ }^{3} \Pi_{2}\left|\mathrm{H}_{\mathrm{SO}}\right|{ }^{5} \Pi_{2}\right\rangle, \\
& =\frac{\sqrt{3}}{2}\left\langle{ }^{3} \Pi_{1}\left|\mathrm{H}_{\text {SO }}\right|^{5} \Pi_{1}\right\rangle,
\end{aligned}
$$

and for the spin-orbit and $L$-uncoupling parameters between the two triplet states $d^{3} \Pi_{g}, v=6$ and $b^{3} \Sigma_{g}^{-}, v=19$

$$
\begin{aligned}
& \left\langle{ }^{3} \Pi_{1}\left|\mathrm{H}_{\mathrm{SO}}\right|^{3} \Sigma_{1}^{-}\right\rangle, \\
& \left\langle{ }^{3} \Pi_{0}\left|\mathrm{H}_{\mathrm{SO}}\right|{ }^{3} \Sigma_{0}^{-}\right\rangle=\sqrt{2}\left\langle{ }^{3} \Pi_{1}\left|\mathrm{H}_{\mathrm{SO}}\right|{ }^{3} \Sigma_{1}^{-}\right\rangle
\end{aligned}
$$

and

$$
\begin{aligned}
& \left\langle{ }^{3} \Pi_{0}\left|\mathrm{BL}_{+}\right|{ }^{3} \Sigma_{1}^{-}\right\rangle, \\
& \left\langle{ }^{3} \Pi_{1}\left|\mathrm{BL}_{+}\right|{ }^{3} \Sigma_{0}^{-}\right\rangle=\sqrt{2}\left\langle{ }^{3} \Pi_{0}\left|\mathrm{BL}_{+}\right|{ }^{3} \Sigma_{1}^{-}\right\rangle, \\
& \left\langle{ }^{3} \Pi_{2}\left|\mathrm{BL}_{+}\right|{ }^{3} \Sigma_{1}^{-}\right\rangle=\sqrt{\frac{x-2}{x}}\left\langle^{3} \Pi_{0}\left|\mathrm{BL}_{+}\right|^{3} \Sigma_{1}^{-}\right\rangle,
\end{aligned}
$$

respectively.

For the Swan-band transition excluding perturbation effects, molecular constants from Tanabashi et al. ${ }^{17}$ are applied. The term energies are defined such that $T=0$ for $v^{\prime \prime}=0$ of the $a^{3} \Pi_{u}$ state yielding the lowest energy level of $-9.8258 \mathrm{~cm}^{-1}$ for $v^{\prime \prime}=0, J^{\prime \prime}=2$, and $N^{\prime \prime}=1$. Absolute energies above the $X^{1} \Sigma_{g}^{+}$ground state of $\mathrm{C}_{2}$ can be obtained by taking into account the difference of 1536.0731 $\mathrm{cm}^{-1}$ reported by Amiot et al. ${ }^{51}$ and considering the different formulation of the effective Hamiltonian by the authors. To obtain the molecular parameters for the $1^{5} \Pi_{g}$, $b^{3} \Sigma_{g}^{-}$states, and their interaction with $d^{3} \Pi_{g}, v=6$, the constants for the unperturbed $d^{3} \Pi_{g}-a^{3} \Pi_{u}$ system are adopted with the exception of the augmented values listed in Table IV (vide infra).

\section{RESULTS}

The middle trace of Fig. 2 shows a DFWM spectrum obtained in the wavelength range of the $v^{\prime}-v^{\prime \prime}=\Delta v=1$ sequence bands of the $d^{3} \Pi_{g}-a^{3} \Pi_{u}$ Swan system. For the applied intensities of a few $\mu \mathrm{J} /$ pulse, an absorptionlike intensity distribution is obtained (see Sec. II). The supersonic expansion from the slit-source produces radicals that are excited to high vibrational levels $\left(T_{\text {vib }}=2000-6000 \mathrm{~K}\right)$ but in each vibrational level a relatively low $J$ population is present $\left(T_{\text {rot }}=50-100 \mathrm{~K}\right)$. The ensuing spectra exhibit, therefore, a reduced number of rotational transitions in each band and a significant simplification of the complex ${ }^{3} \Pi-{ }^{3} \Pi$ transition is achieved. Nonetheless, the spectral features are difficult to assign because multiple sequence bands overlap, i.e., $(6,5),(5,4)$ and $(4,3)$. Furthermore, it has been recognized long ago $^{30}$ that all triplet components of the rotational levels with $N=19$ and 21 and $N \leq 11$ in the $d^{3} \Pi_{g}, v=6$ state are considerably perturbed. The synthetic spectrum (lower trace) is obtained by taking into account line positions and intensities from the PGOPHER program package adopting molecular constants from Tanabashi et al. ${ }^{17}$ For the computation, a vibrational and rotational temperature of 3600 and $50 \mathrm{~K}$, respectively, are assumed and the positions and intensities are convoluted with a Lorentzian line-shape assuming a bandwidth of $0.05 \mathrm{~cm}^{-1}$ (FWHM). As mentioned above, a global analysis of the rotational perturbations has not been achieved and is neglected in the simulations. As a consequence, the experimental DFWM spectrum is poorly reproduced in the lower trace. Significant discrepancies are observed throughout the recorded spectral region. For example, strong transitions between 21420 and $21428 \mathrm{~cm}^{-1}$ appear in the experiment that are not calculated in the simulation. To investigate the involved perturbation effects in detail, an unambiguous assignment of spectral features is required. This task is, however, often not feasible by analyzing one-color spectra such as DFWM, CRD or LIF.

Our recent deperturbation study of the $d^{3} \Pi_{g}, v=4$ state $^{24}$ disclosed the potential of double-resonant four-wave mixing for deperturbation studies of the dicarbon molecule. In spite of the quadratic dependence of the four-wave mixing signal on number density, ${ }^{45}$ the technique is sufficiently sensitive for the detection and characterization of $\mathrm{C}_{2}$ in a molecular beam that is emerging from a discharge slit-jet source. ${ }^{37}$ As for all double-resonance techniques, the use of two distinct input frequencies for TC-RFWM is advantageous for the simplification of spectrally congested regions and the assignment of transitions in general and when perturbation effects are present in particular. A signal is obtained exclusively when both frequencies interact with distinct molecular transitions that share a common level. Straightforward assignments are often possible by tuning one frequency to a known transition and observing probe transitions from the labeled intermediate level. Two typical examples of TC-RFWM spectra are shown in Fig. 3. For the upper trace, intermediate level labeling is achieved by tuning the PUMP laser to coincide with the $\mathrm{R}_{1}(5)$ transition in the $(4,5)$ band at $18319.46 \mathrm{~cm}^{-1}$. Rotational transitions are denoted by ${ }^{\Delta N} \Delta J_{F^{\prime} F^{\prime \prime}}\left(J^{\prime \prime}\right)$, where $N$ is the total angular momentum, $J$, exclusive spin. The spin sublevels, $F_{i}$, for a ${ }^{2 S+1} \Lambda$ state are classified by their energy ordering, i.e., $F_{1}: J=N+S$ for the lowest energy fine structure component and $F_{2 S+1}: J=N-S$ for the highest. For transitions with $\Delta N=\Delta J$ and $F^{\prime}=F^{\prime \prime}$ the rotational notation is simplified to $\Delta J_{F}\left(J^{\prime \prime}\right)$. Thus, by fixing the PUMP laser 


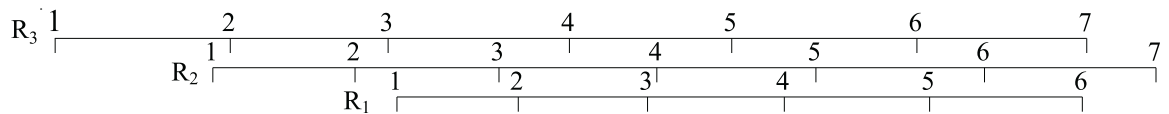

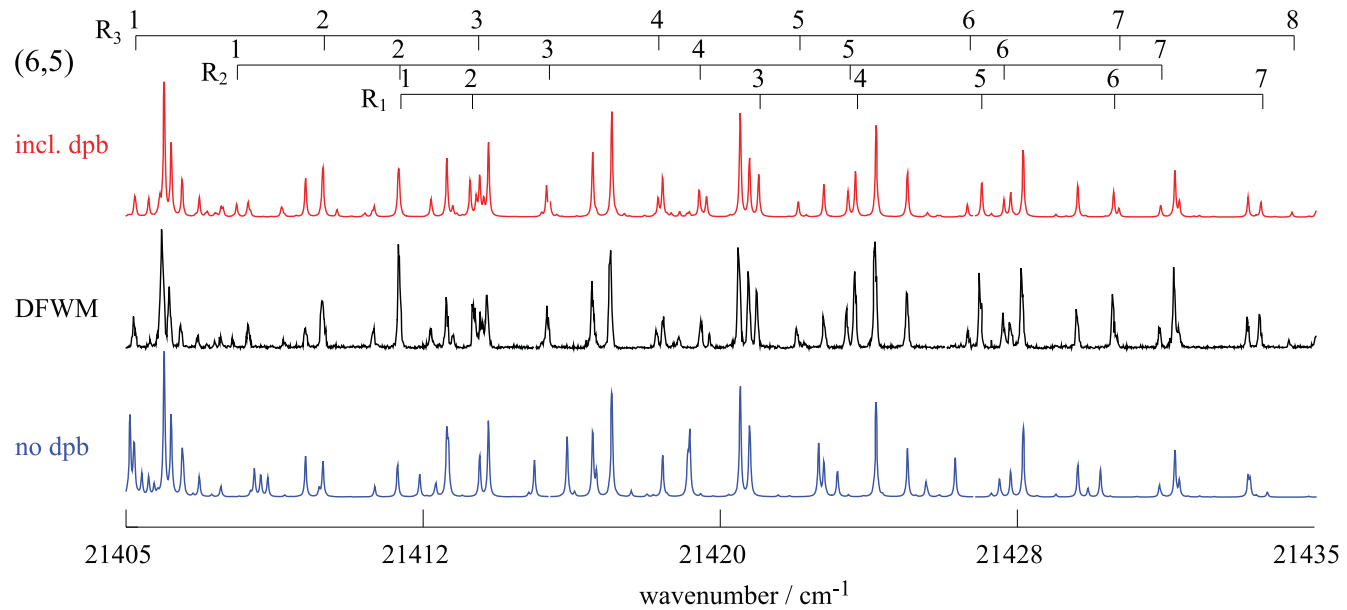

FIG. 2. DFWM spectrum in the wavelength region of the $\Delta v=1$ sequence bands of the $d^{3} \Pi_{g}-a^{3} \Pi_{u}$ electronic system. The bottom trace shows a simulation of the spectral region neglecting perturbations. A comparison with the experimental DFWM shows significant discrepancies. The top trace is a simulation including perturbation effects which has been obtained by the detailed deperturbation analysis in this work. The deperturbed assignments for the three Rsubbands, $\mathrm{R}_{i}(\mathrm{~J})$, are shown on the top for two vibronic bands $\left(v^{\prime}, v^{\prime \prime}\right)$.

wavenumber position to $18319.46 \mathrm{~cm}^{-1}$ and scanning the PROBE laser in the frequency range of the $\Delta v=1$ sequence bands, several intense transitions within the Swan band system are observed. Three UP (or hole-burning) transitions are sharing the common lower state with the PUMP laser $\left(v^{\prime \prime}=5, N^{\prime \prime}=4, J^{\prime \prime}=5, F^{\prime \prime}=1\right)$ : $(6,5) \mathrm{P}_{1}(5),(6,5)$
$\mathrm{Q}_{1}(5)$, and $(6,5) \mathrm{R}_{1}(5)$. In addition, the stimulated emission pumping (SEP) type transition $(4,3) \mathrm{R}_{1}(5)$ is observed sharing the common upper state with the PUMP laser $\left(v^{\prime}=4\right.$, $\left.N^{\prime}=5, J^{\prime}=6, F^{\prime}=1\right)$. For the scans shown in Fig. 3 the transitions within the Swan system are significantly broadened owing to strong saturation $\left(I \approx 1000 I_{\text {sat }}\right)$ in order to

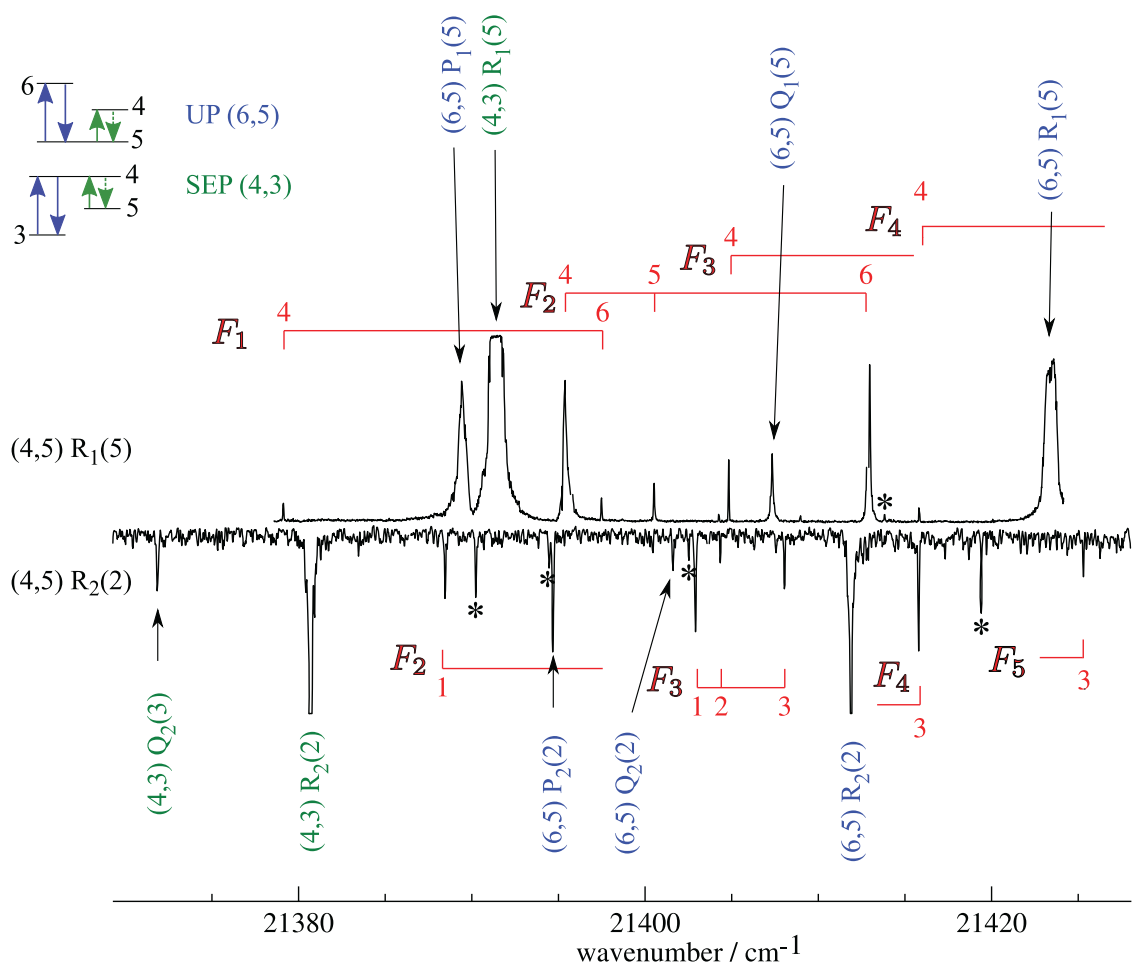

FIG. 3. TC-RFWM in the wavelength region of the $\Delta v=1$ sequence bands of the Swan system. Intermediate level labeling is performed by tuning the PUMP laser to the $\mathrm{R}_{1}(5)$ (upper trace) and $\mathrm{R}_{2}(2)$ (lower trace, inverted) transitions in the $(4,5)$ band, respectively and scanning the PROBE laser near $21400 \mathrm{~cm}^{-1}$. Apart from the intense UP and SEP lines accessed in the $d^{3} \Pi_{g}-a^{3} \Pi_{u}$ system, several transitions from the $a^{3} \Pi_{u}$ state to spin sublevels $\left(F_{i}, i=1 . .5\right)$ of the quintet state are observed. These dark states gain intensity by the electronic interaction of $d^{3} \Pi_{g} \sim 1^{5} \Pi_{g}$. Transitions labeled with asterisks denote weak lines in the Swan band with $\Delta \Omega \pm 1$. See text for details. 
observe transitions to the perturbing states. These weak transitions appear because levels in the vicinity of a perturbation are actually mixed eigenstates containing state character of both levels. Dark states gain thus intensity from bright states and are accessible by TC-RFWM experiments. As explicated in the next section, the spectra unveil the presence of a $1{ }^{5} \Pi_{g}$ state in the vicinity of the $d^{3} \Pi_{g}, v=6$ level. In fact, several UP transitions are observed from the common lower state to different spin sublevels of the $1^{5} \Pi_{g}$ state. For example, the ${ }^{\mathrm{p}} \mathrm{P}_{21}(5),{ }^{\mathrm{q}} \mathrm{Q}_{21}(5)$, and ${ }^{\mathrm{r}} \mathrm{R}_{21}(5)$ transitions excite the $J^{\prime}=4,5$, and 6 rotational states of the $F_{2}$ sublevel $\left(J^{\prime}=N^{\prime}+1\right)$ of the $1^{5} \Pi_{g}$ electronic state. Furthermore, several transitions to the $F_{1}, F_{3}$, and $F_{4}$ sublevels are observed: ${ }^{\circ} \mathrm{P}_{1}(5)$ and ${ }^{\mathrm{q}} \mathrm{R}_{1}(5)$ to $J^{\prime}=4$ and 6 of $F_{1} . J^{\prime}=4$ of $F_{3}$ and $F_{4}$ are excited by ${ }^{\mathrm{q}} \mathrm{P}_{31}(5)$ and ${ }^{\mathrm{r}} \mathrm{P}_{41}(5)$, respectively.

In the lower (inverted) trace, another set of dark states are revealed by the ${ }^{3} \Pi \sim{ }^{5} \Pi$ interaction. Here, the $(4,5) R_{2}(2)$ transition is used for intermediate level labeling by the PUMP laser at $18308.34 \mathrm{~cm}^{-1}$. Apart from two strong SEP and three UP transitions in the Swan system, i.e., $(4,3) \mathrm{Q}_{2}(3),(4,3)$ $\mathrm{R}_{2}(2)$, and $(6,5) \mathrm{P}_{2}(2),(6,5) \mathrm{Q}_{2}(2),(6,5) \mathrm{R}_{2}(2)$, several lines are observed owing to transitions to the $1^{5} \Pi_{g}$ state. $F_{2}(1)$ is clearly visible by the ${ }^{\mathrm{q}} \mathrm{P}_{2}(2)$ transition. $F_{3}(1), F_{3}(2)$ and $F_{3}(3)$ are observed by ${ }^{\mathrm{r}} \mathrm{P}_{32}(2),{ }^{\mathrm{r}} \mathrm{Q}_{32}(2)$, and ${ }^{\mathrm{r}} \mathrm{R}_{32}(2)$, respectively. The $F_{4}(3)$ and $F_{5}(3)$ levels are measured by ${ }^{\mathrm{s}} \mathrm{R}_{42}(2)$ and ${ }^{\mathrm{t}} \mathrm{R}_{52}(2)$, respectively.

Table I lists the wavenumbers of 46 perturbed transitions (fourth column) in the $(6,5)$ and $(6,4)$ bands of the $d^{3} \Pi_{g}-a^{3} \Pi_{u}$ electronic Swan system that have been observed by TC-RFWM and assigned unambiguously (second column) by intermediate level labeling in the $(4,5)$ and $(3,4)$ bands (third column). It is important to notice that several rotational levels of the $d^{3} \Pi_{g}, v^{\prime}=4$ state used for labeling are affected by perturbations owing to the $b^{3} \Sigma_{g}^{-}, v=16$ state as well. Line shifts are taken into account by considering the results of our recent analysis. ${ }^{24}$ Furthermore, most of the characterized energy levels in the upper electronic state $d^{3} \Pi_{g}, v^{\prime}=6$ are accessed from different rotational levels in the lower $a^{3} \Pi_{u}, v=5$ or $v=4$ states. For example, the $F_{1}(3)$ level is measured in all the three branches of the $(6,5)$ band by $\mathrm{P}_{1}(4), \mathrm{Q}_{1}(3)$, and $\mathrm{R}_{1}(2)$, thus further impeding ambiguities due to perturbations that may occur in the lower level. The last column in Table I lists the line shifts of the transitions relative to the simulation model omitting perturbation effects. It is noteworthy that the shifts obtained by performing measurements via different transitions to a specific upper term energy level are in excellent agreement. In the example above, the shifts measured independently in the three branches for $F_{1}(3)$ agree within $0.01 \mathrm{~cm}^{-1}$.

In addition to the measurement of precise line shifts of perturbed transitions, we take advantage of the sensitivity of the technique that allows the observation of the generally weak transitions to the perturbing state (Fig. 3). The inclusion of these extra lines were found to yield significantly modified $B$ and $\lambda$ parameters of the perturbing vibronic state in our recent deperturbation study of the $d^{3} \Pi_{g}, v^{\prime}=4$ state. ${ }^{24}$ Tables II and III list 68 transitions to the $1^{5} \Pi_{g}$ and 8 transitions to the $b^{3} \Sigma_{g}^{-}, v=19$ state, respectively. As for the transitions within the Swan system, term energy levels of
TABLE I. TC-RFWM transitions to $d^{3} \Pi_{g}, v=6\left(\mathrm{~cm}^{-1}\right)$.

\begin{tabular}{|c|c|c|c|c|c|}
\hline $\begin{array}{l}\text { Upper } \\
\text { level }\end{array}$ & Assignment & Intermediate & Observed & Residuals & Perturbation \\
\hline $\mathrm{F} 1(2)$ & $(6,5) \mathrm{pP} 1(3)$ & $(4,5) \mathrm{R} 1(3)$ & 21396.34 & 0.01 & -0.79 \\
\hline $\mathrm{F} 1(2)$ & $(6,5) \mathrm{qQ} 1(2)$ & $(4,5) \mathrm{Q} 1(2)$ & 21404.31 & 0.00 & -0.80 \\
\hline F1(3) & $(6,5) \mathrm{pP} 1(4)$ & $(4,5) \mathrm{R} 1(4)$ & 21393.23 & 0.01 & -1.25 \\
\hline F1(3) & $(6,5) \mathrm{qQ} 1(3)$ & $(4,5) \mathrm{R} 1(3)$ & 21403.97 & 0.01 & -1.25 \\
\hline $\mathrm{F} 1(3)$ & $(6,5) \mathrm{rR} 1(2)$ & $(4,5) \mathrm{Q} 1(2)$ & 21411.95 & 0.00 & -1.26 \\
\hline F1(4) & $(6,5) \mathrm{pP} 1(5)$ & $(4,5) \mathrm{R} 1(5)$ & 21389.51 & -0.03 & -2.36 \\
\hline F1(4) & $(6,5) \mathrm{qQ} 1(4)$ & $(4,5) \mathrm{R} 1(4)$ & 21403.09 & 0.01 & -2.32 \\
\hline F1(4) & $(6,5) \mathrm{rR} 1(3)$ & $(4,5) \mathrm{R} 1(3)$ & 21413.84 & 0.02 & -2.31 \\
\hline $\mathrm{F} 1(5)$ & $(6,5) \mathrm{qQ} 1(5)$ & $(4,5) \mathrm{R} 1(5)$ & 21407.43 & -0.03 & 1.71 \\
\hline $\mathrm{F} 1(5)$ & $(6,5) \mathrm{rR} 1(4)$ & $(4,5) \mathrm{R} 1(4)$ & 21420.98 & -0.02 & 1.71 \\
\hline F1(6) & $(6,5) \mathrm{rR} 1(5)$ & $(4,5) \mathrm{R} 1(5)$ & 21423.51 & 0.03 & 0.97 \\
\hline $\mathrm{F} 1(12)$ & $(6,5) \mathrm{rR} 1(11)$ & $(4,5) \mathrm{R} 1(11)$ & 21444.59 & 0.00 & -1.09 \\
\hline F1(19) & $(6,4) \mathrm{rR} 1(18)$ & $(3,4) R 1(18)$ & 22998.33 & 0.02 & 0.08 \\
\hline $\mathrm{F} 1(20)$ & $(6,4) \mathrm{rR} 1(19)$ & $(3,4) R 1(19)$ & 22999.79 & 0.05 & -3.11 \\
\hline $\mathrm{F} 1(22)$ & $(6,4) \mathrm{rR} 1(21)$ & $(3,4) \mathrm{R} 1(21)$ & 23019.17 & 0.04 & 6.85 \\
\hline $\mathrm{F} 1(23)$ & $(6,4) \mathrm{rR} 1(22)$ & $(3,4) R 1(22)$ & 23016.96 & 0.01 & -0.08 \\
\hline $\mathrm{F} 2(1)$ & $(6,5) \mathrm{pP} 2(2)$ & $(4,5) \mathrm{R} 2(2)$ & 21394.73 & -0.04 & -1.73 \\
\hline $\mathrm{F} 2(1)$ & $(6,5) \mathrm{qQ} 2(1)$ & $(4,5) \mathrm{R} 2(1)$ & 21400.68 & -0.06 & -1.75 \\
\hline $\mathrm{F} 2(2)$ & $(6,5) \mathrm{pP} 2(3)$ & $(4,5) \mathrm{R} 1(3)$ & 21393.00 & -0.04 & -0.70 \\
\hline $\mathrm{F} 2(2)$ & $(6,5) \mathrm{qQ} 2(2)$ & $(4,5) \mathrm{R} 2(2)$ & 21401.78 & -0.02 & -0.68 \\
\hline $\mathrm{F} 2(2)$ & $(6,5) \mathrm{rR} 2(1)$ & $(4,5) \mathrm{R} 2(1)$ & 21407.71 & -0.05 & -0.71 \\
\hline $\mathrm{F} 2(3)$ & $(6,5) \mathrm{pP} 2(4)$ & $(4,5) \mathrm{R} 2(4)$ & 21390.96 & -0.02 & 0.05 \\
\hline $\mathrm{F} 2(3)$ & $(6,5) \mathrm{qQ} 2(3)$ & $(4,5) \mathrm{R} 1(3)$ & 21403.19 & -0.01 & 0.06 \\
\hline $\mathrm{F} 2(3)$ & $(6,5) \mathrm{rR} 2(2)$ & $(4,5) \mathrm{R} 2(2)$ & 21411.95 & -0.01 & 0.06 \\
\hline F2(4) & $(6,5) \mathrm{pP} 2(5)$ & $(4,5) \mathrm{R} 2(5)$ & 21388.65 & 0.03 & 0.33 \\
\hline F2(4) & $(6,5) \mathrm{qQ} 2(4)$ & $(4,5) \mathrm{R} 2(4)$ & 21403.41 & -0.03 & 0.27 \\
\hline F2(4) & $(6,5) \mathrm{rR} 2(3)$ & $(4,5) \mathrm{R} 1(3)$ & 21415.65 & -0.02 & 0.28 \\
\hline $\mathrm{F} 2(5)$ & $(6,5) \mathrm{rR} 2(4)$ & $(4,5) \mathrm{R} 2(4)$ & 21419.55 & 0.00 & 0.35 \\
\hline $\mathrm{F} 2(6)$ & $(6,5) \mathrm{rR} 2(5)$ & $(4,5) \mathrm{R} 2(5)$ & 21423.27 & 0.00 & 0.26 \\
\hline F2(10) & $(6,5) \mathrm{rR} 2(9)$ & $(4,5) \mathrm{R} 2(9)$ & 21438.97 & 0.02 & -0.67 \\
\hline F2(11) & $(6,5) \mathrm{rR} 2(10)$ & $(4,5) \mathrm{R} 2(10)$ & 21444.72 & -0.08 & 0.65 \\
\hline F2(18) & $(6,4) \mathrm{rR} 2(17)$ & $(3,4) \mathrm{R} 2(17)$ & 22999.05 & 0.01 & 1.61 \\
\hline F2(19) & $(6,4) \mathrm{rR} 2(18)$ & $(3,4) \mathrm{R} 2(18)$ & 22999.64 & 0.03 & -2.47 \\
\hline $\mathrm{F} 2(21)$ & $(6,4) \mathrm{rR} 2(20)$ & $(3,4) \mathrm{R} 2(20)$ & 23016.64 & -0.01 & 5.02 \\
\hline F2(22) & $(6,4) \mathrm{rR} 2(21)$ & $(3,4) \mathrm{R} 2(21)$ & 23016.46 & 0.01 & 0.01 \\
\hline $\mathrm{F} 3(0)$ & $(6,5) \mathrm{pP} 3(1)$ & $(4,5) \mathrm{P} 3(1)$ & 21397.61 & -0.01 & 0.82 \\
\hline F3(1) & $(6,5) \mathrm{pP} 3(2)$ & $(4,5) \mathrm{R} 3(2)$ & 21394.29 & 0.00 & 0.97 \\
\hline $\mathrm{F} 3(2)$ & $(6,5) \mathrm{pP} 3(3)$ & $(4,5) \mathrm{R} 3(3)$ & 21391.79 & 0.02 & 1.41 \\
\hline $\mathrm{F} 3(2)$ & $(6,5) \mathrm{rR} 3(1)$ & $(4,5) \mathrm{R} 3(1)$ & 21410.00 & 0.03 & 1.42 \\
\hline $\mathrm{F} 3(3)$ & $(6,5) \mathrm{rR} 3(2)$ & $(4,5) \mathrm{R} 3(2)$ & 21413.89 & -0.01 & 1.44 \\
\hline F3(4) & $(6,5) \mathrm{rR} 3(3)$ & $(4,5) \mathrm{R} 3(3)$ & 21418.48 & 0.06 & 1.61 \\
\hline F3(5) & $(6,5) \mathrm{rR} 3(4)$ & $(4,5) \mathrm{R} 3(4)$ & 21421.98 & -0.02 & 1.20 \\
\hline F3(17) & $(6,4) \mathrm{rR} 3(16)$ & $(3,4) R 3(16)$ & 22997.33 & -0.01 & 0.95 \\
\hline F3(18) & $(6,4) \mathrm{rR} 3(17)$ & $(3,4) \mathrm{R} 3(17)$ & 22999.21 & -0.02 & -2.05 \\
\hline F3(20) & $(6,4) \mathrm{rR} 3(19)$ & $(3,4) R 3(19)$ & 23014.83 & -0.01 & 4.00 \\
\hline F3(21) & $(6,4) \mathrm{rR} 3(20)$ & $(3,4) \mathrm{R} 3(20)$ & 23015.65 & -0.02 & 0.13 \\
\hline
\end{tabular}

the $1^{5} \Pi_{g}$ and $b^{3} \Sigma_{g}^{-}, v=19$ state are generally measured by multiple transitions. For example, the $F_{3}(2)$ level of the quintet state is observed by six independent TC-RFWM transitions applying unique intermediate level labeling schemes. For this measurement perturbation shifts agree within $0.03 \mathrm{~cm}^{-1}$.

Table IV lists the molecular constants including perturbation parameters that are obtained by performing a leastsquares optimization procedure to the 122 listed transitions in Tables I-III using pgopher. ${ }^{50}$ All parameters have been varied 
TABLE II. TC-RFWM transitions to $1^{5} \Pi_{g}\left(\mathrm{~cm}^{-1}\right)$.

\begin{tabular}{|c|c|c|c|c|c|}
\hline $\begin{array}{l}\text { Upper } \\
\text { level }\end{array}$ & Assignment & Intermediate & Observed & Residuals & Perturbation \\
\hline $\mathrm{F} 1(2))$ & $(6,5) \mathrm{pP} 1(3)$ & $(4,5) \mathrm{R} 1(3)$ & 21392.47 & -0.01 & -0.11 \\
\hline $\mathrm{F} 1(2)$ & $(6,5) \mathrm{oP} 12(3)$ & $(4,5) \mathrm{R} 1(3)$ & 21370.01 & 0.00 & -0.11 \\
\hline F1(4) & $(6,5) \mathrm{oP} 1(5)$ & $(4,5) \mathrm{R} 1(5)$ & 21379.12 & 0.00 & -0.25 \\
\hline $\mathrm{F} 1(4)$ & $(6,5) \mathrm{qR} 1(3)$ & $(4,5) \mathrm{R} 1(3)$ & 21403.42 & 0.03 & -0.22 \\
\hline F1(4) & $(6,5) \mathrm{pR} 12(3)$ & $(4,5) \mathrm{R} 1(3)$ & 21380.92 & -0.01 & -0.26 \\
\hline F1(6) & $(6,5) q R 1(5)$ & $(4,5) \mathrm{R} 1(5)$ & 21397.50 & 0.02 & -0.26 \\
\hline $\mathrm{F} 2(0)$ & $(6,5) \mathrm{qP} 23(1)$ & $(4,5) \mathrm{P} 3(1)$ & 21377.46 & 0.00 & -0.83 \\
\hline $\mathrm{F} 2(1)$ & $(6,5) \mathrm{qP} 2(2)$ & $(4,5) \mathrm{R} 2(2)$ & 21388.48 & 0.01 & -1.26 \\
\hline $\mathrm{F} 2(2)$ & $(6,5) \mathrm{qP} 21(3)$ & $(4,5) \mathrm{R} 1(3)$ & 21405.44 & 0.00 & -0.86 \\
\hline $\mathrm{F} 2(2)$ & $(6,5) \mathrm{rQ} 21(2)$ & $(4,5) \mathrm{Q} 1(2)$ & 21413.41 & -0.02 & -0.88 \\
\hline $\mathrm{F} 2(2)$ & $(6,5) \mathrm{pP} 2(3)$ & $(4,5) \mathrm{R} 1(3)$ & 21382.95 & -0.02 & -0.88 \\
\hline $\mathrm{F} 2(2)$ & $(6,5) \mathrm{qR} 23(1)$ & $(4,5) \mathrm{R} 3(1)$ & 21382.17 & 0.01 & -0.85 \\
\hline F2(3) & $(6,5) \mathrm{pP} 21(4)$ & $(4,5) \mathrm{R} 1(4)$ & 21400.53 & -0.01 & 0.18 \\
\hline $\mathrm{F} 2(3)$ & $(6,5) \mathrm{qQ} 21(3)$ & $(4,5) \mathrm{R} 1(3)$ & 21411.27 & -0.01 & 0.18 \\
\hline F2(3) & $(6,5) \mathrm{rR} 21(2)$ & $(4,5) \mathrm{Q} 1(2)$ & 21419.24 & -0.02 & 0.17 \\
\hline $\mathrm{F} 2(3)$ & $(6,5) \mathrm{pQ} 2(3)$ & $(4,5) \mathrm{R} 1(3)$ & 21388.83 & 0.02 & 0.21 \\
\hline F2(4) & $(6,5) \mathrm{pP} 21(5)$ & $(4,5) \mathrm{R} 1(5)$ & 21395.49 & 0.00 & 1.80 \\
\hline F2(4) & $(6,5) \mathrm{qQ} 21(4)$ & $(4,5) \mathrm{R} 1(4)$ & 21409.03 & 0.01 & 1.80 \\
\hline $\mathrm{F} 2(4)$ & $(6,5) \mathrm{rR} 21(3)$ & $(4,5) \mathrm{R} 1(3)$ & 21419.77 & 0.01 & 1.81 \\
\hline $\mathrm{F} 2(5)$ & $(6,5) \mathrm{qQ} 21(5)$ & $(4,5) \mathrm{R} 1(5)$ & 21400.55 & 0.04 & -1.90 \\
\hline $\mathrm{F} 2(5)$ & $(6,5) \mathrm{rR} 21(4)$ & $(4,5) \mathrm{R} 1(4)$ & 21414.09 & 0.03 & -1.90 \\
\hline $\mathrm{F} 2(6)$ & $(6,5) \mathrm{rR} 21(5)$ & $(4,5) \mathrm{R} 1(5)$ & 21412.96 & 0.04 & -0.97 \\
\hline F3(1) & $(6,5) \mathrm{rP} 32(2)$ & $(4,5) \mathrm{R} 2(2)$ & 21403.03 & 0.02 & 2.02 \\
\hline $\mathrm{F} 3(1)$ & $(6,5) \mathrm{sQ} 32(1)$ & $(4,5) \mathrm{R} 2(1)$ & 21408.99 & 0.01 & 2.02 \\
\hline $\mathrm{F} 3(1)$ & $(6,5) \mathrm{qP} 3(2)$ & $(4,5) \mathrm{R} 3(2)$ & 21384.95 & 0.03 & 2.03 \\
\hline F3(2) & $(6,5) \mathrm{rP} 31(3)$ & $(4,5) \mathrm{R} 1(3)$ & 21418.04 & -0.02 & 0.77 \\
\hline $\mathrm{F} 3(2)$ & $(6,5) \mathrm{sQ} 31(2)$ & $(4,5) \mathrm{Q} 1(2)$ & 21426.01 & -0.03 & 0.75 \\
\hline F3(2) & $(6,5) \mathrm{qP} 32(3)$ & $(4,5) \mathrm{R} 1(3)$ & 21395.58 & 0.00 & 0.78 \\
\hline F3(2) & $(6,5) \mathrm{rQ} 32(2)$ & $(4,5) \mathrm{R} 2(2)$ & 21404.34 & -0.01 & 0.77 \\
\hline F3(2) & $(6,5) \mathrm{sR} 32(1)$ & $(4,5) \mathrm{R} 2(1)$ & 21410.29 & -0.03 & 0.76 \\
\hline F3(2) & $(6,5) \mathrm{rR3}(1)$ & $(4,5) \mathrm{R} 3(1)$ & 21394.78 & 0.01 & 0.79 \\
\hline F3(3) & $(6,5) \mathrm{qP} 31(4)$ & $(4,5) \mathrm{R} 1(4)$ & 21411.05 & -0.01 & -0.04 \\
\hline F3(3) & $(6,5) \mathrm{rQ} 31(3)$ & $(4,5) \mathrm{R} 1(3)$ & 21421.76 & -0.03 & -0.06 \\
\hline F3(3) & $(6,5) \mathrm{sR} 31(2)$ & $(4,5) \mathrm{Q} 1(2)$ & 21429.75 & -0.02 & -0.05 \\
\hline F3(3) & $(6,5) \mathrm{qQ} 32(3)$ & $(4,5) \mathrm{R} 1(3)$ & 21399.34 & 0.01 & -0.01 \\
\hline F3(3) & $(6,5) \mathrm{rR3} 2(2)$ & $(4,5) \mathrm{R} 2(2)$ & 21408.09 & 0.00 & -0.03 \\
\hline F3(3) & $(6,5) \mathrm{qR} 3(2)$ & $(4,5) \mathrm{R} 3(2)$ & 21390.00 & 0.00 & -0.03 \\
\hline F3(4) & $(6,5) \mathrm{qP} 31(5)$ & $(4,5) \mathrm{R} 1(5)$ & 21404.90 & -0.01 & -0.30 \\
\hline F3(4) & $(6,5) \mathrm{sR} 31(3)$ & $(4,5) \mathrm{R} 1(3)$ & 21429.14 & -0.04 & -0.33 \\
\hline F3(4) & $(6,5) \mathrm{rR} 32(3)$ & $(4,5) \mathrm{R} 1(3)$ & 21406.72 & 0.01 & -0.28 \\
\hline F3(4) & $(6,5) \mathrm{qR} 3(3)$ & $(4,5) \mathrm{R} 3(3)$ & 21387.69 & 0.00 & -0.29 \\
\hline $\mathrm{F} 3(5)$ & $(6,5) \mathrm{rR3} 2(4)$ & $(4,5) \mathrm{R} 2(4)$ & 21405.16 & 0.01 & -0.34 \\
\hline F3(6) & $(6,5) \mathrm{rR} 32(5)$ & $(4,5) \mathrm{R} 2(5)$ & 21403.53 & 0.03 & -0.30 \\
\hline F4(2) & $(6,5) \mathrm{tQ} 41(2)$ & $(4,5) \mathrm{Q} 1(2)$ & 21435.04 & -0.01 & 0.22 \\
\hline $\mathrm{F} 4(2)$ & $(6,5) \mathrm{rP} 42(3)$ & $(4,5) \mathrm{R} 1(3)$ & 21404.61 & 0.02 & 0.24 \\
\hline $\mathrm{F} 4(2)$ & $(6,5) \mathrm{qP} 43(3)$ & $(4,5) \mathrm{R} 3(3)$ & 21385.60 & 0.02 & 0.25 \\
\hline $\mathrm{F} 4(2)$ & $(6,5) \mathrm{sR} 43(1)$ & $(4,5) \mathrm{R} 3(1)$ & 21403.82 & 0.04 & 0.27 \\
\hline F4(3) & $(6,5) \mathrm{qP} 42(4)$ & $(4,5) \mathrm{R} 2(4)$ & 21394.87 & -0.01 & -0.03 \\
\hline $\mathrm{F} 4(3)$ & $(6,5) \mathrm{sR} 42(2)$ & $(4,5) \mathrm{R} 2(2)$ & 21415.89 & 0.01 & -0.02 \\
\hline F4(3) & $(6,5) \mathrm{rR} 43(2)$ & $(4,5) \mathrm{R} 3(2)$ & 21397.78 & 0.00 & -0.03 \\
\hline F4(4) & $(6,5) \mathrm{rP} 41(5)$ & $(4,5) \mathrm{R} 1(5)$ & 21415.83 & -0.02 & -0.24 \\
\hline F4(4) & $(6,5) \mathrm{qP} 42(5)$ & $(4,5) \mathrm{R} 2(5)$ & 21390.63 & 0.02 & -0.20 \\
\hline F4(4) & $(6,5) \mathrm{sR} 42(3)$ & $(4,5) \mathrm{R} 1(3)$ & 21417.64 & -0.02 & -0.24 \\
\hline F4(4) & $(6,5) \mathrm{rR} 43(3)$ & $(4,5) \mathrm{R} 3(3)$ & 21398.67 & 0.03 & -0.19 \\
\hline $\mathrm{F} 4(5)$ & $(6,5) \mathrm{sR} 42(4)$ & $(4,5) \mathrm{R} 2(4)$ & 21418.84 & -0.01 & -0.31 \\
\hline $\mathrm{F} 4(6)$ & $(6,5) \mathrm{sR} 42(5)$ & $(4,5) \mathrm{R} 2(5)$ & 21419.61 & 0.01 & -0.27 \\
\hline $\mathrm{F} 4(12)$ & $(6,5) \operatorname{tR} 41(11)$ & $(4,5) \mathrm{R} 1(11)$ & 21446.77 & -0.07 & 0.84 \\
\hline F5(3) & $(6,5) \mathrm{rP} 52(4)$ & $(4,5) \mathrm{R} 2(4)$ & 21404.35 & 0.00 & -0.24 \\
\hline F5(3) & $(6,5) \operatorname{tR} 52(2)$ & $(4,5) \mathrm{R} 2(2)$ & 21425.33 & -0.01 & -0.25 \\
\hline
\end{tabular}

TABLE II. (Continued).

\begin{tabular}{lccccc}
\hline \hline $\begin{array}{r}\text { Upper } \\
\text { level }\end{array}$ & Assignment & Intermediate & Observed & Residuals & Perturbation \\
\hline F5(3) & $(6,5) \mathrm{sR} 53(2)$ & $(4,5) \mathrm{R} 3(2)$ & 21407.26 & 0.01 & -0.23 \\
F5(4) & $(6,5) \mathrm{rP52}(5)$ & $(4,5) \mathrm{R} 2(5)$ & 21403.02 & -0.01 & -0.62 \\
F5(4) & $(6,5) \mathrm{sQ52}(4)$ & $(4,5) \mathrm{R} 2(4)$ & 21417.86 & 0.01 & -0.60 \\
F5(4) & $(6,5) \mathrm{tR} 52(3)$ & $(4,5) \mathrm{R} 1(3)$ & 21430.08 & 0.00 & -0.61 \\
F5(4) & $(6,5) \mathrm{sR} 53(3)$ & $(4,5) \mathrm{R} 3(3)$ & 21411.09 & 0.03 & -0.58 \\
F5(5) & $(6,5) \mathrm{sR} 53(4)$ & $(4,5) \mathrm{R} 3(4)$ & 21412.05 & 0.01 & -0.48 \\
F5(6) & $(6,5) \mathrm{tR} 52(5)$ & $(4,5) \mathrm{R} 2(5)$ & 21437.52 & 0.02 & -0.44 \\
F5(10) & $(6,5) \operatorname{tR} 52(9)$ & $(4,5) \mathrm{R} 2(9)$ & 21442.86 & -0.04 & 0.55 \\
F5(11) & $(6,5) \mathrm{tR} 52(10)$ & $(4,5) \mathrm{R} 2(10)$ & 21440.05 & 0.04 & -0.88 \\
\hline \hline
\end{tabular}

simultaneously until the best fit possible has been reached. An excellent agreement between the observed transitions and the simulation is obtained. The root mean square value for the reduction is $0.024 \mathrm{~cm}^{-1}$ which is approximately half of the specified laser bandwidth of the dye lasers. Precise experimentally determined molecular constants for the $1{ }^{5} \Pi_{g}$ are reported for the first time to the best of our knowledge. The centrifugal distortion constant $D$ is not accessible owing to the low $J$ quantum numbers of the measured transitions. The same reason prevents the accurate evaluation of the $\Lambda$-doubling parameters $p$ and $q$. It is worth mentioning that for quintet states the higher order spin-spin term $\theta$ has to be considered. However, an improvement of the fit has not been achieved by the inclusion of this term in the Hamiltonian. This finding is anticipated by taking into account the small value for the first order spin-spin parameter $\lambda$. The results from a configuration interaction study by Kirby and $\mathrm{Liu}^{31}$ are listed for comparison. The authors predicted the $1^{5} \Pi_{g}$ valence state among others with a bond energy of $1.70 \mathrm{eV}$ and calculated the origin, $T_{e}$, with an estimated accuracy of $\approx 2700 \mathrm{~cm}^{-1}$ which has been determined by comparison with experimentally measured valence states. The theoretically calculated rotational constant is about $10 \%$ smaller than the experimentally determined value. The good agreement with the computed ab initio values suggests that the vibrational ground state of the $1^{5} \Pi_{g}$ is observed. However, a definitive assignment of the vibrational state is not directly accessible from the rotational analysis of this single band system.

TABLE III. TC-RFWM transitions to $b^{3} \Sigma_{g}^{-}, v=19\left(\mathrm{~cm}^{-1}\right)$.

\begin{tabular}{lccccc}
\hline \hline $\begin{array}{l}\text { Upper } \\
\text { level }\end{array}$ & Assignment & Intermediate & Observed & Residuals & Perturbation \\
\hline F1(18) & $(6,4)$ qR12(17) & $(3,4) \mathrm{R} 2(17)$ & 22994.35 & 0.02 & -0.19 \\
F1(20) & $(6,4) \mathrm{rR} 1(19)$ & $(3,4) \mathrm{R} 1(19)$ & 23031.07 & -0.03 & 3.10 \\
F1(22) & $(6,4) \mathrm{rR} 1(21)$ & $(3,4) \mathrm{R} 1(21)$ & 22996.06 & -0.02 & -6.80 \\
F2(17) & $(6,4) \mathrm{qR} 23(16)$ & $(3,4) \mathrm{R} 3(16)$ & 22991.48 & -0.01 & 0.17 \\
F2(19) & $(6,4) \mathrm{rR} 2(18)$ & $(3,4) \mathrm{R} 2(18)$ & 23027.49 & 0.01 & 2.56 \\
F2(21) & $(6,4) \mathrm{rR} 2(20)$ & $(3,4) \mathrm{R} 2(20)$ & 22995.21 & 0.01 & -4.91 \\
F3(18) & $(6,4) \mathrm{rR} 3(17)$ & $(3,4) \mathrm{R} 3(17)$ & 23024.97 & 0.01 & 2.22 \\
F3(20) & $(6,4) \mathrm{rR} 3(19)$ & $(3,4) \mathrm{R} 3(19)$ & 22994.31 & 0.00 & -3.84 \\
\hline \hline
\end{tabular}


TABLE IV. Optimized molecular constants for the $1^{5} \Pi_{g}, d^{3} \Pi_{g}, v=6$, and the $b^{3} \Sigma_{g}^{-}, v=19$ states. All values are in $\mathrm{cm}^{-1}$. The origin, $\mathrm{T}$ is relative to the $a^{3} \Pi_{u}, v=0$ level. Numbers in parenthesis are one standard deviation.

\begin{tabular}{|c|c|c|c|}
\hline State & Parameter & Value & Reference \\
\hline \multirow[t]{5}{*}{$1^{5} \Pi_{g}$} & $\mathrm{~T}$ & $29258.5922(48)$ & $29941.99^{b}$ \\
\hline & $\mathrm{B}$ & $1.14413(11)$ & $1.012^{\mathrm{b}}$ \\
\hline & A & $8.9450(47)$ & \\
\hline & $\lambda$ & $-0.0428(23)$ & \\
\hline & o & $-0.0744(39)$ & \\
\hline \multirow[t]{4}{*}{$b^{3} \Sigma_{g}^{-}, v=19$} & $\mathrm{~T}$ & $29442.1348(843)$ & $29434.25^{c}$ \\
\hline & B & $1.179368(214)$ & $1.178804^{\mathrm{c}}$ \\
\hline & $\mathrm{D} \times 10^{6}$ & $6.5066^{\mathrm{c}}$ & $6.5066^{\mathrm{c}}$ \\
\hline & $\lambda$ & $0.142(22)$ & $0.1548^{\mathrm{c}}$ \\
\hline \multirow[t]{4}{*}{$d^{3} \Pi_{g}, v=6$} & $\mathrm{~T}$ & $29259.3736(32)$ & $29259.704(14)^{\mathrm{a}}$ \\
\hline & A & $-12.8223(90)$ & $-13.082(35)^{\mathrm{a}}$ \\
\hline & $\mathrm{p}$ & $0.00467(91)$ & $0.00104(65)^{\mathrm{a}}$ \\
\hline & $\mathrm{q}$ & $-0.000964(44)$ & $-0.001514(17)^{\mathrm{a}}$ \\
\hline$\left\langle d^{3} \Pi_{1}, v=6\left|H_{s o}\right| 1^{5} \Pi_{1}\right\rangle$ & & $4.6220(88)$ & \\
\hline$\left\langle d^{3} \Pi_{1}, v=6\left|H_{s o}\right| b^{3} \Sigma_{1}^{-}, v=19\right\rangle$ & & $0.7855(110)$ & \\
\hline$\frac{1}{\sqrt{x}}\left\langle d^{3} \Pi_{0}, v=6\left|B L_{+}\right| b^{3} \Sigma_{1}^{-}, v=19\right\rangle$ & & $0.31192(37)$ & \\
\hline
\end{tabular}

a Reference 17.

${ }^{\mathrm{b}}$ Listed values are for $v=0$ in Ref. 31 .

${ }^{\mathrm{c}}$ Extrapolated from the results in Ref. 51.

Similar to the $1^{5} \Pi_{g}$, no experimentally determined molecular constants are available for the $b^{3} \Sigma_{g}^{-}, v=19$ state. The results can be compared with extrapolated values on the basis of the work by Amiot et al. ${ }^{51}$ for the Ballik-Ramsay system $\left(b^{3} \Sigma_{g}^{-}-a^{3} \Pi_{u}\right)$ where 13 bands between $(0,0)$ and $(6,3)$ have been investigated. Owing to the low $J$ quantum numbers measured in this study, the centrifugal distortion constant, $D$ has been fixed at the extrapolated value. An overall good agreement of the experimental values for this "dark" state with the extrapolation is obtained, in particular, when considering that the Dunham expansion coefficients are determined for relatively low vibrational levels only $\left(v^{\prime} \leq 6\right)$. A similar agreement with the extrapolated values has been obtained for $v^{\prime}=16$ in the deperturbation study of $d^{3} \Pi_{g}, v=4,{ }^{24}$ further indicating the consistency of the results obtained by applying the TC-RFWM technique.

The global fit to the measured transitions is significantly improved by floating the origin, $T$, spin-orbit, $A$, and the $\Lambda$ doubling constants, $p$ and $q$, of the upper Swan level $d^{3} \Pi_{g}, v=6$. The average error of the fit is reduced from 0.189 to $0.024 \mathrm{~cm}^{-1}$. The obtained origin, $T$ and spin-orbit, $A$ are in good agreement with the computed values from the equilibrium constants reported by Tanabashi et al. ${ }^{17}$ In fact, the spectroscopic parameters obtained by Fourier transform spectroscopy for $v=6$ were not used to derive the equilibrium molecular constants for the $d^{3} \Pi_{g}$ state owing to the extensive perturbation affecting the accuracy of the experimental precision. However, equilibrium constants were determined by performing a fit to the Dunham expansion using the relatively unperturbed vibrational levels $v=1,2,3,5,7$, and 8. By applying the reported coefficients, the origin and spinorbit parameters for the unperturbed $v=6$ level are estimated as 29259.211 and $-12.740 \mathrm{~cm}^{-1}$, respectively. The values obtained in this study for $T$ and $A$ are in significantly better agreement with this assessment than the previous constants (fourth row in Table IV), strongly supporting the appropriate deperturbation of this vibrational level.

The results are further sustained by adopting the new molecular constants in Table IV and computing the residuals of 67 "unperturbed" line positions with $2 \leq N \leq 27$ in the $(6,5)$ and $(6,8)$ bands reported by Tanabashi et al. ${ }^{17}$ The average error of these transitions is reduced by roughly a factor 3 to $0.040 \mathrm{~cm}^{-1}$. Similar accuracies are computed for their transitions in the relatively unperturbed vibrational bands. The deperturbation analysis clearly shows that perturbation affects a large number of these transitions. Computing residuals by taking into account the deperturbed constants for the $d^{3} \Pi_{g}, v=6$ state and neglecting the spin-orbit interaction with the $1^{5} \Pi_{g}$ state and, in addition, the $L$-uncoupling and spin-orbit interactions with the $b^{3} \Sigma_{g}^{-}, v=19$ state yields a dramatically increased average error of $0.263 \mathrm{~cm}^{-1}$ thus indicating that significant perturbation-induced line shifts occur.

The term energies for the $d^{3} \Pi_{g}, v=6,1{ }^{5} \Pi_{g}$ and $b^{3} \Sigma_{g}^{-}, v=19$ states shown in Fig. 4 are evaluated from the observed transitions by subtracting the lower state rotational energies calculated by adopting the molecular constants of the $a^{3} \Pi_{u}$ state from Tanabashi. ${ }^{17}$ The value of $1.59984 J(J+1)$ $\mathrm{cm}^{-1}$ has been subtracted from the energies in order to display the the significant parts of the vibronic levels and their crossings. The solid lines are calculated by the model described above and adopting the molecular constants listed in Table IV. It is evident why the deperturbation of $d^{3} \Pi_{g}, v=6$ is so difficult. Below $J \approx 15$, the three spin sublevels of the $d$ state are affected by the five $F$-levels of the quintet. At higher $J$ values, each sublevel is crossed by the three energy term curves of the $b^{3} \Sigma_{g}^{-}, v=19$ state. Thus, many rotational levels in this range are affected by perturbations. The strongest interactions occur at rotational quantum numbers close to a term energy curve crossing. For example, the sublevel $F_{4}$ of the quintet state intersects $F_{1}$ of the $d^{3} \Pi_{g}, v=6$ at $J=12$. The 

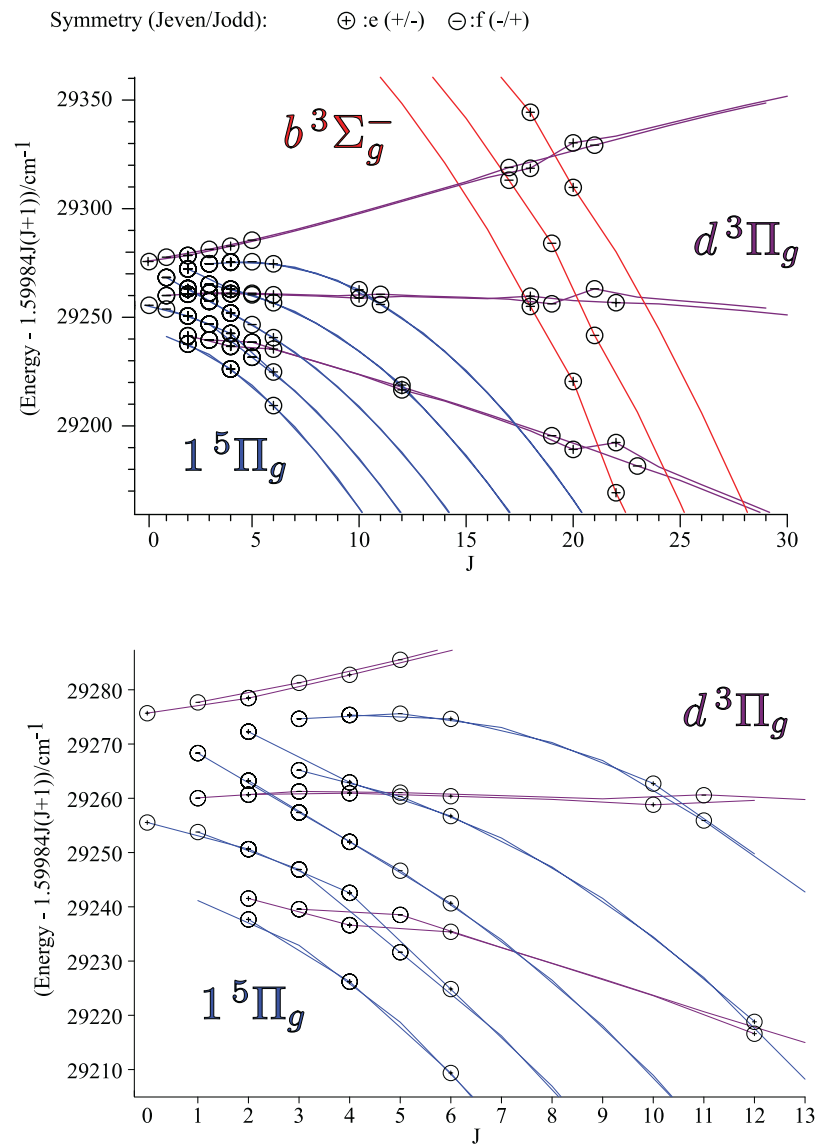

FIG. 4. Observed term energies of the $d^{3} \Pi_{g}, v=6,1^{5} \Pi_{g}$ and $b^{3} \Sigma_{g}^{-}$, $v=19$ states vs rotational quantum number $J$. (a) The solid lines are computed term values by taking into account the deperturbed molecular constants listed in Table IV. Parities are denoted by $\oplus$ and $\ominus$ signs for e and $\mathrm{f}$ levels, respectively. (b) shows an expanded view of the $d^{3} \Pi_{g}, v=6 \sim 1{ }^{5} \Pi_{g}$ interaction. See text for details.

unperturbed energies of the two rotational levels at the crossing point would be separated by $\approx 0.25 \mathrm{~cm}^{-1}$ only. However, spin-orbit interaction shifts each level in opposite direction by roughly $1 \mathrm{~cm}^{-1}$. Even larger shifts occur by $L$-uncoupling and spin-orbit interactions for $b^{3} \Sigma_{g}^{-} \sim d^{3} \Pi_{g}$. For instance, the crossing of the $F_{1}$ term energy curves shifts each $F_{1}(22)$ level by $\approx 6.8 \mathrm{~cm}^{-1}$.

The term energy shifts are depicted in Fig. 5 by plotting the deviations of the rotational level energies of the $d^{3} \Pi_{g}, v=6$ state determined in this study from the simulated values obtained from the molecular constants omitting perturbation effects. ${ }^{17}$ Perturbations owing to two electronic states are clearly visible. On one hand, strong shifts are observed for $J=4,5$, and 12 in the $F_{1}$ sublevel and for $J=10$ and 11 in $F_{2}$. For these perturbations both, $e$ and $f$ parity terms are affected simultaneously which is expected for a ${ }^{5} \Pi_{g}$ perturber state. On the other hand, substantial perturbations at $N=19$ and 21 exhibit clear propensities for a specific parity level in each $F$ sublevel. The latter finding is in accordance with a perturbation by the $b^{3} \Sigma_{g}^{-}$state because for this state only odd- $N$ rotational levels are allowed by nuclear spin-statistics. For example, for the $F_{1}$ component only $e$ parity levels are affected by the inhomogeneous $L$-uncoupling $(\Delta \Omega \pm 1)$ and the homogeneous spin-orbit $(\Delta \Omega=0)$ interac-

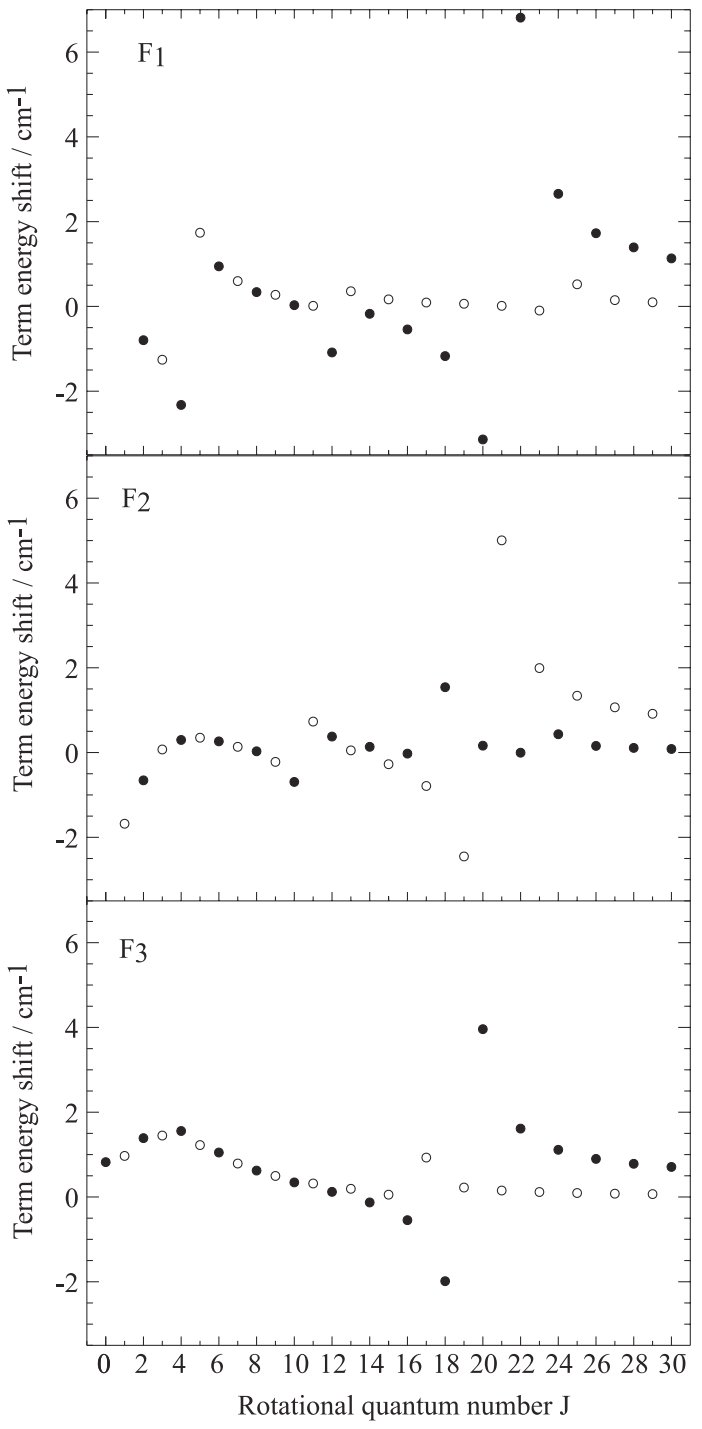

FIG. 5. Shifts of the perturbed rotational term energies in the $d^{3} \Pi_{g}, v=6$ state plotted against the upper state rotational quantum number $J^{\prime}$ for the three $F$ components. Filled and open circles denote $e$ and $f$ parity, respectively. See text for details.

tions because for the $F_{1}$ sublevels $(J=N+1)$ of the $b^{3} \Sigma_{g}^{-}$ state solely $e$ parity levels exist.

\section{DISCUSSION}

Initial attempts to analyze the complex perturbations occurring for rotational states with $N \leq 11$ in the $d^{3} \Pi_{g}, v=6$ state were unsuccessful. Considering that inversion symmetry rules precludes $g \sim u$ perturbations, the five nearby-lying electronic states shown in Fig. 6 were taken into account as potential perturbers. The previously suggested $B^{1} \Delta_{g}$ state by Meinel and Messerle ${ }^{30}$ (vide supra) was ruled out by energy considerations. The Fourier transform emission spectroscopy of the $B^{1} \Delta_{g}-A^{1} \Pi_{u}$ transition ${ }^{21}$ for $v^{\prime}=0-5$ allows a relatively accurate extrapolation up to $v^{\prime}=15$ by taking into account the equilibrium constants of this well behaved electronic state. In fact, recent deperturbation studies on $v=2$ of the $d^{3} \Pi_{g}$ state in our laboratory have shown that the $B^{1} \Delta_{g}, v=9$ is crossing the $F_{1}$ and $F_{2}$ levels of the $d$ state 
$1^{5} \Pi_{g}$

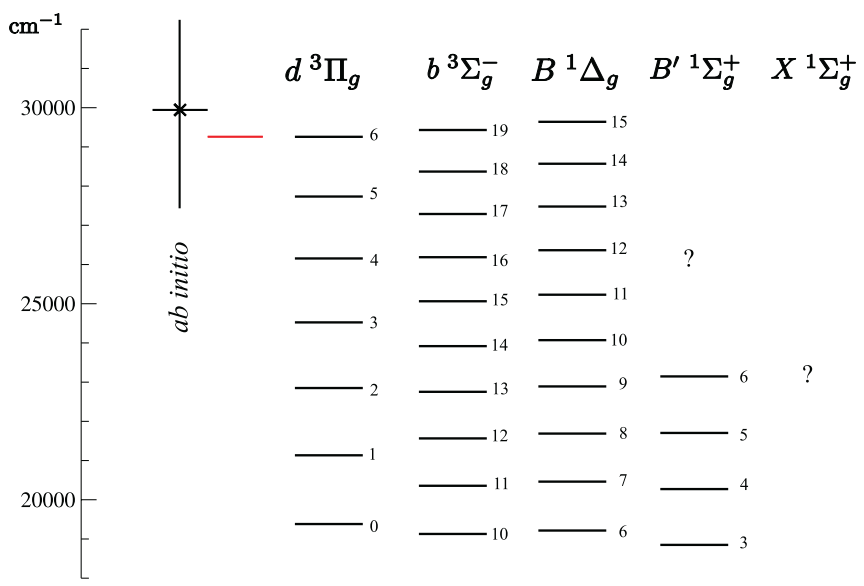

FIG. 6. Close lying vibronic levels with an inversion symmetry equivalent to the $d^{3} \Pi_{g}$ state that may interact by spin-orbit or $L$-uncoupling mechanisms. Vibrational quantum numbers are indicated on the right-hand side. The origins of the $b$ and $B$ states are computed by adopting equilibrium constants determined for $v \leq 6$ and 5, respectively. An accurate extrapolation to high-lying vibrational levels of the $B^{\prime}$ and ground state $X$ from $v \leq 3$ and 6 , respectively, is not feasible. See text for details.

near $J=15$ and 9, respectively, which is in favorable agreement with the extrapolated term energy curve of the $B$ state. A similar accordance is obtained for $v=0$ and 1 . Thus, rotational perturbations in the $d^{3} \Pi_{g}, v=6$ by the $B^{1} \Delta_{g}, v=15$ should occur at $J$ levels around 30 . Furthermore, by considering the selection rule $\Delta \Omega=0$ for a spin-orbit interaction, the crossing of the single term energy curve of the $B$ state $(\Omega=2)$ would affect a few rotational levels mainly in the $F_{1}$ and $F_{2}$ sublevels of the $d$ state and yield a much simpler perturbation pattern.

Unfortunately, an accurate Dunham extrapolation for the remaining two singlet states in question, i.e., $X^{1} \Sigma_{g}^{+}$and $B^{\prime}{ }^{1} \Sigma_{g}^{+}$, to higher vibrational levels is not feasible. It has been recognized $^{21,31}$ that both states interact and mutually distort their potential energy curves. The computed potentials nearly blend at an equilibrium distance of $\approx 1.6 \AA$ yielding to anomalous shapes as a function of $r$. The equilibrium constants obtained for the $B^{\prime 21}$ and $X$ states $^{52}$ for $v$ up to 3 and 6 , respectively, are thus not suitable for an extrapolation to higher vibrational quanta. However, a perturbation by the ${ }^{1} \Sigma_{g}^{+}$states can be ruled out by symmetry considerations. Owing to nuclear spin statistics only even (e-parity) $J$ levels exist in a ${ }^{1} \Sigma_{g}^{+}$state. These states can interact with even $J$ levels only which is in contrast to the observed term energy shifts of both, $e$ and $f$ parity levels (Fig. 5). Lastly, as mentioned in the introduction and confirmed by this work, the $b^{3} \Sigma_{g}^{-}, v=19$ state perturbs rotational levels with $N=19$ and 21 as measured by Meinel and Messerle ${ }^{30}$ and confirmed by Tanabashi and co-workers. ${ }^{17}$ Thus, this state cannot give reason for the observed perturbations for $N \leq 11$ of the $d^{3} \Pi_{g}, v=6$ level. Little and Brown ${ }^{29}$ proposed on the basis of the computed potential energy curves by Kirby and $\mathrm{Liu}^{31}$ a perturbation by the energetically lowest quintet state, $1^{5} \Pi_{g}$, to rationalize the low $J$ shifts. In consideration of this assumption, crossing points on the $F_{1}$ term energy curve (Fig. 4 ) were identified by the substantial shifts of rotational levels that have been assigned unquestionably by intermediate level labeling. Initial attempts to model the quintet state with a negative spin-orbit constant, $A$, in accordance with the ${ }^{3} \Pi$-states of the dicarbon resulted in large residuals for perturbed rotational transitions to the $F_{2}$ and $F_{3}$ sublevels of the $d^{3} \Pi_{g}, v=6$ state. The average error of the fit is dramatically reduced by taking the opposite sign. As indicated above, further improvements of the fit are achieved by optimizing the molecular constants of four parameters characterizing the $d^{3} \Pi_{g}, v=6$ level as listed in Table IV. Thus, the elimination of the known neighboring electronic states as potential perturbers of the $d^{3} \Pi_{g}, v=6$ state at low $J$ values and the excellent fit (vide supra) to the 122 observed perturbed and extra line positions strongly support the presence of the $1^{5} \Pi_{g}$ high-spin state.

Likewise, assignment by double-resonance spectroscopy proved advantageous for the analysis of the $b^{3} \Sigma_{g}^{-}, v=19$ $\sim d^{3} \Pi_{g}, v=6$ interaction occurring between $N=13$ and 27. Attempts to estimate the perturbation by considering the reported positions of Tanabashi ${ }^{17}$ and Meinel ${ }^{30}$ failed due to erroneous assignments in the complex and overlapping spectrum. In fact, strong perturbation effects scramble the characteristic triplet structure of consecutive $R_{3}, R_{2}$, and $\mathrm{R}_{1}$ transitions in the Swan band and hinder correct assignments. To disentangle the complicated spectral features, an initial guess of the term energy curve crossings (Fig. 4) has been obtained by considering less perturbed lines only that are assignable in a straightforward manner. The positions of strongly perturbed transitions and their extra lines were subsequently predicted by the guess parameters of the model and accurately determined by applying intermediate level labeling.

A comparison of our line positions and assignments in the $(6,5)$ band imply that the $(6,5) R_{2}(17), R_{3}(17), R_{1}(18)$ and $\mathrm{R}_{2}$ (18) reported by Meinel and Messerle ${ }^{30}$ should be reassigned to $R_{1}(18), R_{2}(17), R_{2}(18)$ and $R_{3}(17)$, respectively. In addition, the assignment of $\mathrm{R}_{1}(21)$ and $\mathrm{R}_{1}(22)$ should be exchanged. With these modifications, an average error of 0.043 $\mathrm{cm}^{-1}$ is obtained for the 45 transitions measured by Meinel and Messerle and adopting the parameters listed in Table IV. The excellent agreement with their measured line positions is further supporting the correct deperturbation analysis of this band.

Numerous perturbed transitions listed in Table I have also been reported by Tanabashi et al. ${ }^{17}$ and are in general agreement. However, spectral assignment by intermediate level labeling yields for $\mathrm{P}_{1}(5)$ line position in the $(6,5)$ band 21389.51 $\mathrm{cm}^{-1}$ instead of $21391.79 \mathrm{~cm}^{-1}$. In addition, our value for $\mathrm{R}_{1}(5)$ at $21423.51 \mathrm{~cm}^{-1}$ is $1.51 \mathrm{~cm}^{-1}$ higher. Furthermore, the measurements suggest that the positions of the $(6,5) R_{2}(17)$ and $R_{1}(18)$ should be interchanged. In fact, excellent accuracies of roughly $0.04 \mathrm{~cm}^{-1}$ are obtained for a subset of 151 perturbed line positions reported in the $(6,5)$ and $(6,8)$ bands for $N \leq 27$ which are compared with model calculations adopting the constants in Table IV. However, large discrepancies for numerous remaining transitions suggest incorrect assignments. A possible explanation might be the complex Fourier transform spectrum where multiple sequence bands overlap (Sec. IV) rendering assignments of perturbed transitions ex- 
tremely difficult. The TC-RFWM technique is advantageous to eliminate these ambiguities.

In the discussion above, the crossing of the $1^{5} \Pi_{g}$ with $d^{3} \Pi_{g}, v=6$ leading to the enhanced emission (HP bands), as suggested by Little and Browne ${ }^{29}$ is verified. The perturbation is characterized in detail and exactly calculable. The occurrence of the large and level-specific interaction elucidates the nonthermal population distribution in the $v=6$ level that is observed in numerous environments at different pressures and temperatures. The mechanism involved has been put forth by Gelbart and Freed ${ }^{53}$ who realized that intrinsic intramolecular interactions such as perturbations between electronic states can have an important effect on the pressure dependent details of the luminescence behavior of small molecules. The model is particularly important for the spin-forbidden electronic transition that is investigated in the present work where the direct electronic inelastic process is significantly less probable. ${ }^{54}$ In this "gateway" model the cross sections for collision-induced transitions between two different electronic states, $\mathrm{E}$ and $\mathrm{E}^{\prime}$, are

$$
\begin{aligned}
\sigma_{E, J ; E^{\prime}, J^{\prime}} \simeq & \sigma_{E, J ; E, J^{\prime}} C_{E, E^{\prime}}\left(J^{\prime}\right)^{2} \\
& +\sigma_{E^{\prime}, J ; E^{\prime}, J^{\prime}} C_{E, E^{\prime}}(J)^{2},
\end{aligned}
$$

where $\sigma_{E, J ; E, J^{\prime}}$ and $\sigma_{E^{\prime}, J ; E^{\prime}, J^{\prime}}$ are the $J \rightarrow J^{\prime}$ purely rotationchanging cross-sections within the $\mathrm{E}$ and $\mathrm{E}^{\prime}$ electronic states, respectively, and $C_{E, E^{\prime}}$ is the isolated-molecule $\mathrm{E}, J \sim \mathrm{E}^{\prime}, J$ mixing coefficient. If only few vibronic levels are significantly mixed they should act as a "doorway" or "gateway" through which all population flows from one electronic state to the other. In the following we show qualitative evidence that gateway states are essential to rationalize the observed emission anomalies of the HP bands. In this respect, the curve crossing of the $F_{1}$ component of the $d^{3} \Pi_{g}, v=6$ with $F_{4}$ of the $1^{5} \Pi_{g}$ state is of particular interest (Fig. 4). The energy separation of the unperturbed levels at $J=12$ is only $0.28 \mathrm{~cm}^{-1}$. Spin-orbit interaction between the electronic states shifts the two levels $1.93 \mathrm{~cm}^{-1}$ apart. Note however, the unperturbed $F_{1}(12)$ is not a pure $\Omega$ state but due to the spin-orbit interaction $(A \hat{\mathbf{L}} \cdot \hat{\mathbf{S}})[\mathrm{Eq} .(1)]$ an admixture of the nominal $d^{3} \Pi_{2 g}(49 \%), d^{3} \Pi_{1 g}(41 \%)$, and $d^{3} \Pi_{0 g}(10 \%)$ levels. Upon perturbation by the $1^{5} \Pi_{g}$ the resulting $F_{1}(12)$ state reduces its $d^{3} \Pi_{g}$-character to $53 \%$, i.e., $d^{3} \Pi_{2 g}(28 \%), d^{3} \Pi_{1 g}(21 \%)$, and $d^{3} \Pi_{0 g}(4 \%)$ and gains $47 \%$ $1^{5} \Pi_{g}$-character, i.e., $1^{5} \Pi_{1 g}(7 \%), 1^{5} \Pi_{0 g}(16 \%), 1^{5} \Pi_{1 g},(1 \%)$ $1{ }^{5} \Pi_{2 g}(7 \%)$, and $1{ }^{5} \Pi_{3 g}(16 \%)$. Figure 7 depicts the fractional $1^{5} \Pi_{g}$-character gain of perturbed $d^{3} \Pi_{g}, v=6$ rotational terms for the three $F$ components. Clearly, $F_{1}(12)$ and to a lesser extend $F_{1}(4), F_{1}(5), F_{2}(1)$, and $F_{2}(2)$ have to be considered as "gateway" states. Thus, if the chemical reactions mentioned in the introduction populate the metastable $1{ }^{5} \Pi_{g}$ state, perturbation-facilitated transfer to the $d^{3} \Pi_{g}$, $v=6$ can occur and promote enhanced emission in the Swan system. It is interesting to mention that the HP bands are characterized by a double-headed spectral feature. ${ }^{16,27,35,55,56}$ The "gateway" states mentioned above readily rationalize the observation. The band head of $v=6$ is formed by closelyspaced rotational levels of the P-branches. Enhanced emission is observed by the nonthermal population of the low $J$

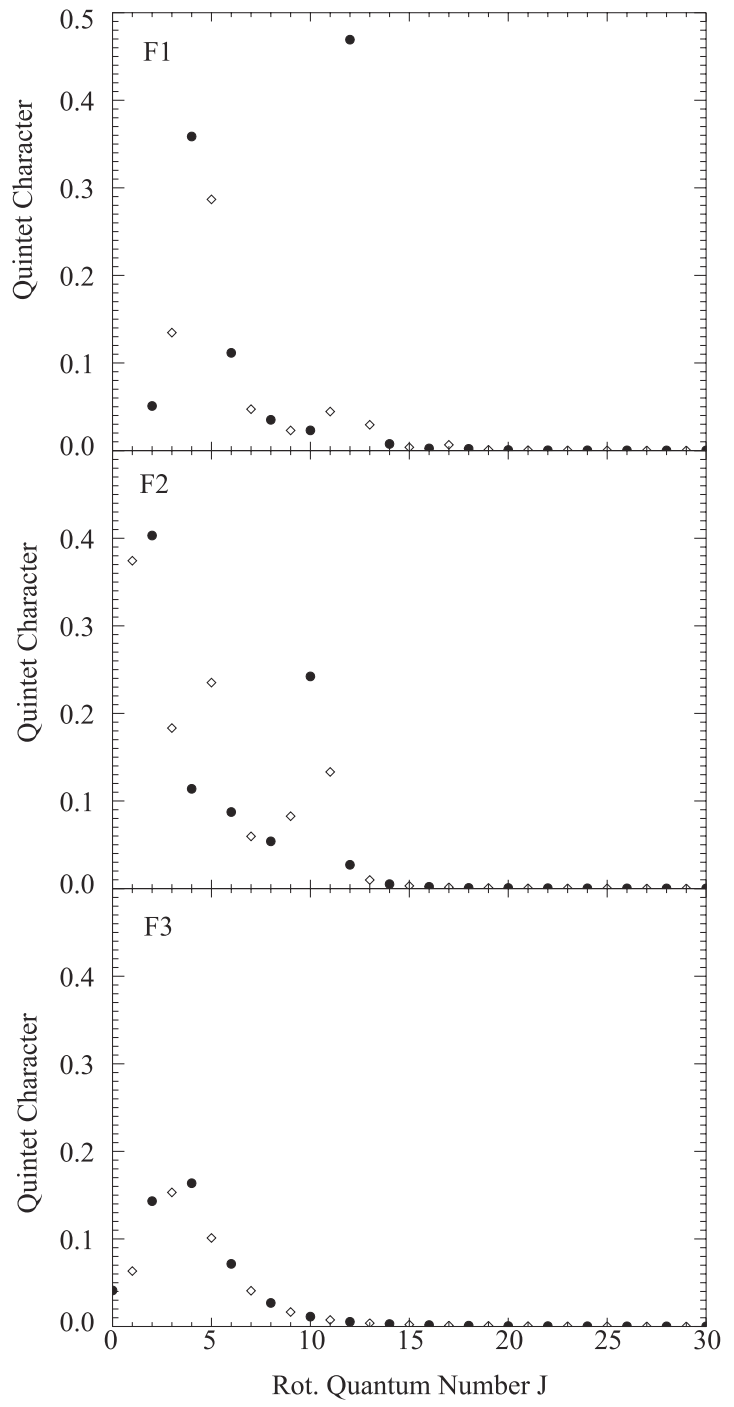

FIG. 7. $1^{5} \Pi_{g}$-character of perturbed $d^{3} \Pi_{g}, v=6$ rotational energy terms. Strongly mixed eigenfunctions are "gateway" states through which population flows from one electronic state to the other.

"gateway" states. In addition, a second "head" is formed that originates mainly from the strongly mixed $F_{1}(12)$ increasing the emission in the $\mathrm{R}$-branch via $\mathrm{R}_{1}(11)$. A detailed quantitative analysis of the population transfer between the $d^{3} \Pi_{g}$ and $1^{5} \Pi_{g}$ is under way in our laboratories and will be the subject of a forthcoming publication.

\section{CONCLUSION}

Double-resonant four-wave mixing experiments have been applied for a deperturbation study of the $d^{3} \Pi_{g}, v=6$ state of $\mathrm{C}_{2}$. Rotational levels with $N \leq 11$ are perturbed by a high-spin state which is experimentally identified for the first time. The vibronic origin, rotational, spin-orbit, spin-spin constants, and the $\Lambda$-doubling parameter of the $1^{5} \Pi_{g}$ are accurately determined. In addition, curve crossing at $N=19$ and 21 with the $b^{3} \Sigma_{g}^{-}, v=19$ state is quantitatively evaluated and yield origin, rotational and spin-spin constants of the perturber state. Results are obtained by performing intermediate level labeling for unambiguous assignment of perturbed 
and perturbing transitions. The sensitivity of the method allows the observation of 122 transitions including 76 lowintensity excitations to "dark states" that are made visible by electronic state-mixing. Considering strongly mixed "gateway" states, we conclude that population flow from a chemically produced $1^{5} \Pi_{g}$ state to the $d^{3} \Pi_{g}, v=6$ is the origin of the nonthermal emission which is observed in numerous environments. Furthermore, the identified "gateways" between the quintet and triplet state rationalize the double-headed spectral feature of the high-pressure bands.

\section{ACKNOWLEDGMENTS}

This work is supported by the Swiss NSF-CH (200020_124542/1) and the Swiss Federal Office of Energy (100708). The help of Patrick Fehlmann performing measurements is greatly acknowledged.

${ }^{1}$ P. Rousselot, S. M. Hill, M. H. Burger, D. A. Brain, C. Laffont, and G. Moreels, Icarus 146, 263 (2000).

${ }^{2}$ R. I. Kaiser, Chem. Rev. 102, 1309 (2002).

${ }^{3}$ K. Nyholm, M. Kaivola, and C. Aminoff, Opt. Commun. 107, 406 (1994).

${ }^{4}$ P. E. Bengtsson and M. Alden, Appl. Phys. B 60, 51 (1995).

${ }^{5}$ C. Kaminski and P. Ewart, Appl. Phys. B 61, 585 (1995).

${ }^{6}$ C. F. Kaminski, I. G. Hughes, and P. Ewart, J Chem. Phys. 106, 5324 (1997)

${ }^{7}$ J. B. Wills, J. A. Smith, W. E. Boxford, J. M. F. Elks, M. N. R. Ashfold, and A. J. Orr-Ewing, J. Appl. Phys. 92, 4213 (2002).

${ }^{8}$ A. Staicu, R. L. Stolk, and J. J. ter Meulen, J. Appl. Phys. 91, 969 (2002).

${ }^{9}$ G. P. Smith, C. Park, J. Schneiderman, and J. Luque, Combust. Flame 141, 66 (2005).

${ }^{10} \mathrm{X}$. Mercier, E. Therssen, J. Pauwels, and P. Desgroux, Proc. Combust. Inst. 30, 1655 (2005).

${ }^{11}$ A. Goldman and S. Cheskis, Appl. Phys. B 92, 281 (2008).

${ }^{12}$ C. V. V. Prasad and P. F. Bernath, Astrophys. J. 426, 812 (1994).

${ }^{13}$ W. H. Wollaston, Philos. Trans. R. Soc. London 92, 365 (1802).

${ }^{14}$ W. Swan, Ann. Phys. Chem. 176, 306 (1857).

${ }^{15}$ J. G. Phillips and S. P. Davis, The Swan System of the C2 Molecule: The Spectrum of the HgH Molecule (University of California, Berkeley and Los Angeles, 1968).

${ }^{16}$ A. Tanabashi and T. Amano, J. Mol. Spectrosc. 215, 285 (2002).

${ }^{17}$ A. Tanabashi, T. Hirao, T. Amano, and P. F. Bernath, Astrophys. J., Suppl. Ser. 169, 472 (2007).

${ }^{18}$ E. A. Ballik and D. A. Ramsay, Astrophys. J. 137, 61 (1963).

${ }^{19}$ J. Callomon and A. C. Gilby, Can. J. Phys. 41, 995 (1963).

${ }^{20}$ J. G. Phillips, J. Mol. Spectrosc. 28, 233 (1968).
${ }^{21}$ M. Douay, R. Nietmann, and P. Bernath, J. Mol. Spectrosc. 131, 261 (1988).

${ }^{22}$ C. Amiot, Astrophys. J., Suppl. Ser. 52, 329 (1983).

${ }^{23}$ J. A. Joester, M. Nakajima, N. J. Reilly, D. L. Kokkin, K. Nauta, S. H. Kable, and T. W. Schmidt, J. Chem. Phys. 127, 214303 (2007).

${ }^{24}$ P. Bornhauser, G. Knopp, T. Gerber, and P. Radi, J. Mol. Spectrosc. 262, 69 (2010).

${ }^{25}$ J. G. Fox and G. Herzberg, Phys. Rev. 52, 638 (1937).

${ }^{26}$ G. Herzberg, Astrophys. J. 89, 290 (1939).

${ }^{27}$ A. Fowler, Mon. Not. R. Astron. Soc. 70, 484 (1910).

${ }^{28}$ R. C. Johnson and R. K. Asundi, Proc R. Soc. London 124, 668 (1929).

${ }^{29}$ C. E. Little and P. G. Browne, Chem. Phys. Lett. 134, 560 (1987).

${ }^{30} \mathrm{H}$. Meinel and G. Messerle, Astrophys J. 154, 381 (1968).

${ }^{31}$ K. Kirby and B. Liu, J. Chem. Phys. 70, 893 (1979).

${ }^{32}$ C. Kunz, P. Harteck, and S. Dondes, J. Chem. Phys. 46, 4157 (1967).

${ }^{33}$ D. W. Naegeli and H. B. Palmer, J. Mol. Spectrosc. 26, 152 (1968).

${ }^{34}$ F. Gosse, N. Sadeghi, and J. C. Pebay-Peyroula, Chem. Phys. Lett. 13, 557 (1972).

${ }^{35}$ P. Caubet and G. Dorthe, Chem. Phys. Lett. 218, 529 (1994).

${ }^{36}$ M. Tulej, M. Meisinger, G. Knopp, A. M. Walser, T. Gerber, and P. P. Radi, J. Raman Spectrosc 38, 1022 (2007).

${ }^{37}$ F. J. Mazzotti, E. Achkasova, R. Chauhan, M. Tulej, P. P. Radi, and J. P. Maier, Phys. Chem. Chem Phys. 10, 136 (2008).

${ }^{38}$ M. Tulej, G. Knopp, T. Gerber, and P. P. Radi, J Raman Spectrosc. 41, 853 (2010).

${ }^{39}$ H. Linnartz, Phys. Scr. 69, C37 (2004).

${ }^{40}$ Y. Prior, Appl. Opt. 19, 1741 (1980).

${ }^{41}$ P. P. Radi, H. M. Frey, B. Mischler, A. P. Tzannis, P. Beaud, and T. Gerber, Chem. Phys. Lett. 265, 271 (1997).

${ }^{42}$ R. L. Abrams and R. C. Lind, Opt. Lett. 2, 94 (1978).

${ }^{43}$ S. Williams, R. N. Zare, and L. A. Rahn, J. Chem. Phys. 101, 1072 (1994).

${ }^{44}$ S. Williams, R. N. Zare, and L. A. Rahn, J. Chem. Phys. 101, 1093 (1994).

${ }^{45}$ S. Williams, E. A. Rohlfing, L. A. Rahn, and R. N. Zare, J. Chem. Phys. 106, 3090 (1997).

${ }^{46}$ G. Lloyd and Bratfalean, J. Opt. Soc. Am. B 16, 952 (1999).

${ }^{47}$ G. M. Lloyd and P. Ewart, J. Chem. Phys. 110, 385 (1999).

${ }^{48}$ T. A. Reichardt and R. P. Lucht, J. Chem. Phys. 111, 10008 (1999).

${ }^{49}$ J. M. Brown and A. J. Merer, J. Mol. Spectrosc. 74, 488 (1979).

${ }^{50}$ C. M. Western, PGOPHER, A Program for Simulating Rotational Structure (2009).

${ }^{51}$ C. Amiot, J. Chauville, and J. P. Maillard, J. Mol. Spectrosc. 75, 19 (1979).

${ }^{52}$ M. Chan, S. Yeung, Y. Wong, Y. Li, W. Chan, and K. Yim, Chem. Phys. Lett. 390, 340 (2004).

${ }^{53}$ W. M. Gelbart and K. F. Freed, Chem. Phys. Lett. 18, 470 (1973).

${ }^{54} \mathrm{H}$. Lefebvre-Brion and R. W. Field, The Spectra and Dynamics of Diatomic Molecules, Rev. and enlarged ed. (Elsevier, Amsterdam, 2004).

${ }^{55}$ R. W. B. Pearse and A. G. Gaydon, The Identification of Molecular Spectra, 4th ed. (Chapman and Hall, London, 1976).

${ }^{56}$ W. Faust, L. Goldberg, B. Craig, and R. Weiss, Chem. Phys. Lett. 83, 265 (1981). 